\title{
Parton intrinsic motion in inclusive particle production: unpolarized cross sections, single spin asymmetries and the Sivers effect
}

\author{
Umberto D'Alesio* and Francesco Murgia ${ }^{\dagger}$ \\ Dipartimento di Fisica, Università di Cagliari and \\ Istituto Nazionale di Fisica Nucleare, Sezione di Cagliari \\ Casella Postale n. 170, I-09042 Monserrato (CA), Italy
}

(Dated: October 16, 2018)

\begin{abstract}
The relevance of intrinsic (or primordial) transverse momentum of partons in the inclusive production of particles at high energy and moderately large $p_{T}$ has been known for a long time, beginning with Drell-Yan and diphoton processes, and continuing with photon and meson production in hadronic collisions. In view of its renewed interest in the context of polarized processes and single spin asymmetries we perform, in the framework of perturbative QCD with the inclusion of spin and $\boldsymbol{k}_{\perp}$ effects, a detailed analysis of several such processes in different kinematical situations. We show that the inclusion of these effects leads, at the level of accuracy reachable in this approach, to an overall satisfactory agreement between theoretical predictions and experimental unpolarized data, thus giving support to the study of spin effects and single spin asymmetries within the same scheme. We present results for transverse single spin asymmetries, generated by the so-called Sivers effect, in inclusive pion and photon production in proton-proton collisions. We compare our results with the available experimental data and with previous results obtained using simplified versions of this approach.

PACS numbers: 12.38.Bx, 13.88.+e, 13.85.Ni, 13.85.Qk
\end{abstract}

\section{INTRODUCTION}

It has been known for a long time that the intrinsic (or primordial) transverse momentum of partons inside hadrons involved in high-energy processes may play a relevant role. A typical example is the Drell-Yan process, where the primordial, non-perturbative transverse momentum of the initial partons is directly related to the lower part of the transverse momentum spectrum of the observed lepton pair. A similar example is diphoton production in hadronic collisions. Since the first applications of perturbative quantum chromodynamics (pQCD) to inclusive particle production in hadronic collisions, the role of these effects has been of some interest and several generalizations of the usual collinear pQCD approach have been presented [1] (throughout this paper, by collinear pQCD we mean the approach in which intrinsic transverse momentum, $\boldsymbol{k}_{\perp}$, effects are integrated out up to a factorization scale and all partons/hadrons are assumed to be collinear with parent hadrons/partons). In recent years, several papers have reconsidered these effects, since collinear pQCD, even at next-to-leading order (NLO) seems to underestimate experimental results for photon and pion production in hadronic collisions in the central rapidity and moderately large $p_{T}$ region [2-4]. It was found that the inclusion of intrinsic transverse momentum effects allows in most cases to reconcile theoretical calculations with experimental results. This requires a relatively large average transverse momentum, showing some dependence on the c.m. energy of the process considered. This probably indicates that some effects due to higher order pQCD corrections are effectively embodied in the intrinsic momentum contributions, a point which needs to be further clarified.

Almost independently of the above mentioned studies, the role of intrinsic transverse momentum has received a lot of attention in the context of polarization effects in inclusive particle production at high energy and moderately large $p_{T}$, in particular regarding transverse single spin asymmetries (SSA). Contrary to former expectations of pQCD [5], there are several experimental observations showing that SSA can be large in this kinematical regime. It was originally suggested by Sivers [6] that pQCD with the inclusion of transverse momentum effects in parton distribution functions could be able to explain these results. Sivers' suggestion has been further extended in a number of subsequent papers $[7,8]$. There are several alternative possible explanations for SSA, basically non-perturbative in nature. Recently, Bourrely and Soffer [9] have claimed that most of the experimental data on SSA cannot be explained by pQCD, on the basis that the collinear approach fails to reproduce the corresponding unpolarized cross sections (which enter

\footnotetext{
*Electronic address: umberto.dalesio@ca.infn.it
}

${ }^{\dagger}$ Electronic address: francesco.murgia@ca.infn.it 
the denominator of the SSA) by 1-2 orders of magnitude. As a matter of fact, in all previous papers on SSA using pQCD-based approaches, a detailed analysis of unpolarized cross sections was not performed. The main reason for this was that a number of effects (scale dependences, NLO corrections) might cancel out in the SSA, which are ratios of (polarized) cross sections. Moreover, data on unpolarized cross sections in the kinematical regions where SSA are measured to be relevant are scarce. Considering the increasing theoretical and experimental interest in high-energy spin effects, a detailed combined analysis of unpolarized cross sections and SSA, within the same pQCD approach, is timely and worthwhile.

Based on these considerations, in this paper we first perform a detailed analysis of a large amount of data on unpolarized cross sections for inclusive particle production in several processes and in different kinematical situations. Let us stress that our approach and aim are different in many respects from (and complementary to) those in Ref.s [2-4]: our main interest remains the study of SSA. Most of the interesting results on SSA are in kinematical regions different from those preferentially considered in the above mentioned papers, that is at $1 \mathrm{GeV} / c \lesssim p_{T} \lesssim 3 \mathrm{GeV} / c$ and large $\left|x_{F}\right|=2\left|p_{L}\right| / \sqrt{s}$. Moreover, calculations of SSA are at present limited to the inclusion of leading-order contributions. Therefore, in this paper we limit ourself to study unpolarized cross sections at the same level of accuracy. In fact, our goal is not that of reproducing with very high precision the experimental results on unpolarized cross sections. Rather, we want to show that the same approach used for SSA, at the same level of accuracy, is able to reproduce unpolarized cross sections for several processes and different kinematical situations, up to a reasonable factor (of the order of 2-3) which may be attributed to NLO corrections (the so-called $K$-factors), scale dependences, etc., which we expect, at least, to partly cancel out in the SSA.

To this aim, we use here a generalization of the usual collinear pQCD approach to inclusive particle production with the inclusion of spin and transverse momentum effects. We will take into account $\boldsymbol{k}_{\perp}$ effects for all partons involved in the process, including the fragmentation process where required, which has been neglected in most of the Ref.s [2-4]. This considerably improves the treatment of $\boldsymbol{k}_{\perp}$ effects with respect to Ref.s [7, 10], where a simplified approach was adopted, including $\boldsymbol{k}_{\perp}$ effects only at first non-vanishing order and with a simplified partonic kinematics. Therefore, in this paper we will reconsider SSA for several processes already analyzed in Ref. [7], comparing the results obtained in the two approaches.

The plan of the paper is the following: in section II we summarize our approach, which will be largely utilized in the rest of the paper. In section III we present and discuss our results concerning the unpolarized cross sections for the Drell-Yan process and for inclusive pion and photon production in hadronic collisions. A detailed comparison with available experimental results in several kinematical configurations is given. In section IV we then present our results for SSA in inclusive pion and photon production in hadronic collisions, including only the so-called Sivers effect [6-8] as the possible mechanism for generating the SSA. Our conclusions are presented in section V; the Appendix collects useful relations regarding the kinematics.

\section{PHENOMENOLOGICAL APPROACH}

In this section we present the basic ideas, ingredients and formulae of our approach; details on kinematics are given in the Appendix. Let us consider the inclusive production of large $p_{T}$ hadrons in high-energy hadron collisions, that is the process $A B \rightarrow C X$. The starting point of our approach is the well-known collinear pQCD factorized expression for the corresponding differential cross section:

$$
\frac{E_{C} d \sigma^{A B \rightarrow C X}}{d^{3} \boldsymbol{p}_{C}}=\sum_{a, b, c, d} \int d x_{a} d x_{b} d z f_{a / A}\left(x_{a}, Q^{2}\right) f_{b / B}\left(x_{b}, Q^{2}\right) \frac{d \hat{\sigma}^{a b \rightarrow c d}}{d \hat{t}} \frac{\hat{s}}{\pi z^{2}} \delta(\hat{s}+\hat{t}+\hat{u}) D_{C / c}\left(z, Q^{2}\right) .
$$

Here $x_{a}, x_{b}(z)$ are the light-cone momentum fractions of partons $a, b$ (hadron $C$ ) with respect to the parent hadrons $A, B$ (parton $c) ; f\left(x, Q^{2}\right), D\left(z, Q^{2}\right)$ are the well-known non-perturbative parton distribution functions (PDF) and fragmentation functions (FF) respectively, where the intrinsic transverse momentum dependence has been integrated over up to the factorization scale $Q^{2}$. Furthermore, $\hat{s}, \hat{t}, \hat{u}$ are the Mandelstam variables for the partonic scattering process, $a b \rightarrow c d$, with all (massless) partons taken to be collinear with the corresponding hadrons. The Dirac delta function accounts for energy-momentum conservation in the elementary process and allows to express e.g. $z$ as a function of $x_{a}$ and $x_{b}$ and the hadronic Mandelstam invariants (see also the Appendix).

Extending this formalism with the inclusion of intrinsic transverse momentum of partons is not trivial at all and poses several problems. To start with, a complete formal proof of the factorization theorem is still missing in this case. The validity of factorization has been conjectured by Collins [11] and proved only for the Drell-Yan process and in $e^{+} e^{-}$annihilation [12]. Quite recently a factorization formula for semi-inclusive deep inelastic scattering in the current fragmentation region has been proved [13]. A formally complete definition of transverse-momentum dependent 
PDF's and FF's and of their evolution properties is still missing. In a partonic approach and at leading twist in the factorization scale $Q$ the usual collinear parton densities $f_{a / A}\left(x_{a}, Q^{2}\right)$ are simply generalized to transverse-momentum dependent distributions $\hat{f}_{a / A}\left(x_{a}, \boldsymbol{k}_{\perp a}, Q^{2}\right)$, where $\boldsymbol{k}_{\perp a}$ is the transverse momentum of parton $a$ with respect to the light-cone direction of hadron $A$, such that

$$
f_{a / A}\left(x_{a}, Q^{2}\right)=\int d^{2} \boldsymbol{k}_{\perp a} \hat{f}_{a / A}\left(x_{a}, \boldsymbol{k}_{\perp a}, Q^{2}\right) .
$$

Analogously, the usual parton fragmentation function $D_{C / c}\left(z, Q^{2}\right)$ generalizes to $\hat{D}_{C / c}\left(z, \boldsymbol{k}_{\perp C}, Q^{2}\right)$, where $\boldsymbol{k}_{\perp C}$ is the transverse momentum of the final hadron $C$ with respect to the light-cone direction of the fragmenting parton $c$, and

$$
D_{C / c}\left(z, Q^{2}\right)=\int d^{2} \boldsymbol{k}_{\perp C} \hat{D}_{C / c}\left(z, \boldsymbol{k}_{\perp C}, Q^{2}\right) .
$$

Intrinsic transverse momentum effects are of higher twist; consistently, a complete higher-twist treatment of the process would be required. However, we are far from being able to perform this complete analysis, since it introduces new, unknown non-perturbative PDF's and FF's and quark-gluon correlations; furthermore, the usual partonic interpretation of PDF's and FF's would be lost in this case.

Despite these problems, there is a strong phenomenological evidence that transverse momentum effects in the partonic kinematics and dynamics are very relevant and may contribute to account for several experimental results for (un)polarized cross sections in inclusive particle production, difficult to explain in the collinear pQCD approach.

This is why this generalized approach has been extensively employed in recent years; some of the resulting phenomenological outcomes are quite promising, although much work remains to be done on its formal aspects. This leads to a modified expression for the differential cross section, Eq. (1), which now reads

$$
\begin{aligned}
\frac{E_{C} d \sigma^{A B \rightarrow C X}}{d^{3} \boldsymbol{p}_{C}} & =\sum_{a, b, c, d} \int d x_{a} d^{2} \boldsymbol{k}_{\perp a} d x_{b} d^{2} \boldsymbol{k}_{\perp b} d z d^{3} \boldsymbol{k}_{C} \delta\left(\boldsymbol{k}_{C} \cdot \hat{\boldsymbol{p}}_{c}\right) \hat{f}_{a / A}\left(x_{a}, \boldsymbol{k}_{\perp a}, Q^{2}\right) \hat{f}_{b / B}\left(x_{b}, \boldsymbol{k}_{\perp b}, Q^{2}\right) \\
& \times \frac{\hat{s}}{x_{a} x_{b} s} \frac{d \hat{\sigma}^{a b \rightarrow c d}}{d \hat{t}}\left(x_{a}, x_{b}, \hat{s}, \hat{t}, \hat{u}\right) \frac{\hat{s}}{\pi} \delta(\hat{s}+\hat{t}+\hat{u}) \frac{1}{z^{2}} J\left(z,\left|\boldsymbol{k}_{C}\right|\right) \hat{D}_{C / c}\left(z, \boldsymbol{k}_{C}, Q^{2}\right) .
\end{aligned}
$$

Notice that the partonic cross sections $d \hat{\sigma} / d \hat{t}$ depend, via the Mandelstam variables, on the intrinsic transverse momenta of partons. Notice also that the integration over the transverse momentum of the observed hadron $C$ with respect to the light-cone direction of the fragmenting parton $c$ runs over a generic three-momentum $\boldsymbol{k}_{C}$, defined in the c.m. of the initial hadrons $A$ and $B$ (we will call this the hadronic c.m. frame in the following); the delta function $\delta\left(\boldsymbol{k}_{C} \cdot \hat{\boldsymbol{p}}_{c}\right)$ guarantees that $\boldsymbol{k}_{C}$ is in fact always orthogonal to $\boldsymbol{p}_{c}$, the parton $c$ three-momentum, and also the correct normalization of the fragmentation function, according to Eq. (3), which makes $\boldsymbol{k}_{C}$ completely equivalent to $\boldsymbol{k}_{\perp C}$. The extra phase-space factor $J\left(z,\left|\boldsymbol{k}_{C}\right|\right) / z^{2}$ is the proper invariant Jacobian factor for the transformation from the parton momentum $\boldsymbol{p}_{c}$ to the hadron momentum $\boldsymbol{p}_{C}$ with inclusion of transverse momentum effects; the term $\hat{s} /\left(x_{a} x_{b} s\right)$ restores proper flux factor for non-collinear (in the hadronic c.m. frame) parton-parton collision (see Appendix).

Let us stress at this point that, once collinear pQCD is complemented with the inclusion of $\boldsymbol{k}_{\perp}$ effects, not only Eq. (1) modifies to Eq. (4), but additional contributions to the unpolarized cross section are in principle possible [14-16]. These contributions to the unpolarized cross section could play a relevant role when considering asymmetries with respect to some measurable azimuthal angle related to the partonic process (e.g. in the Drell-Yan process, see [15]); however, we have explicitly checked that they give negligible contributions to the cross section for the inclusive process $A B \rightarrow C X$, where all "internal", partonic variables are integrated over. Therefore, they are of little relevance for this analysis, and will not be further considered in the rest of the paper. A more complete discussion will be given elsewhere [16].

Eq. (4) has been widely used in the literature, starting from the pioneering work of Feynman, Field and Fox [1]. Several papers have shown in the recent past that intrinsic $\boldsymbol{k}_{\perp}$ 's are indeed necessary in order to improve the agreement between next-to-leading order (NLO) pQCD calculations and experimental data for inclusive pion and photon production at high energies and moderately large $p_{T}[2-4]$.

An expression similar to Eq. (4) holds also for the numerator of a SSA, $\left(d \sigma^{\uparrow}-d \sigma^{\downarrow}\right) /\left(d \sigma^{\uparrow}+d \sigma^{\downarrow}\right) \propto d \Delta^{N} \sigma / d \sigma$, replacing, for the polarized particles involved, the corresponding unpolarized PDF, FF and partonic cross sections by the appropriate polarized ones, $\Delta^{N} f, \Delta^{N} D, d \Delta^{N} \hat{\sigma}$ (see Ref. [10] and section III for more details). At leading twist there are four such new spin and $\boldsymbol{k}_{\perp}$ dependent functions to take into account:

$$
\begin{aligned}
\Delta^{N} f_{a / A^{\uparrow}} & \equiv \hat{f}_{a / A^{\uparrow}}\left(x, \boldsymbol{k}_{\perp}\right)-\hat{f}_{a / A^{\downarrow}}\left(x, \boldsymbol{k}_{\perp}\right) ; \quad \Delta^{N} f_{a^{\uparrow} / A} \equiv \hat{f}_{a^{\uparrow} / A}\left(x, \boldsymbol{k}_{\perp}\right)-\hat{f}_{a \downarrow / A}\left(x, \boldsymbol{k}_{\perp}\right) ; \\
\Delta^{N} D_{C / c^{\uparrow}} & \equiv \hat{D}_{C / c^{\uparrow}}\left(z, \boldsymbol{k}_{\perp}\right)-\hat{D}_{C / c^{\downarrow}}\left(z, \boldsymbol{k}_{\perp}\right) ; \Delta^{N} D_{C^{\uparrow} / c} \equiv \hat{D}_{C^{\uparrow} / c}\left(z, \boldsymbol{k}_{\perp}\right)-\hat{D}_{C^{\downarrow} / c}\left(z, \boldsymbol{k}_{\perp}\right),
\end{aligned}
$$


two in the PDF sector, Eq. (5), and two in the FF sector, Eq. (6); the first functions in Eq.s (5), (6) are respectively the so-called Sivers [6] and Collins [11] function. The second ones are respectively the function introduced by Boer and Mulders [14, 15] and the so-called "polarizing" FF [14, 17]. For a general overview on spin and $\boldsymbol{k}_{\perp}$-dependent PDF's and FF's see also Ref. [18].

Regarding SSA, in this paper we will focus on the Sivers effect in inclusive pion and photon production. For a phenomenological study of the role of the Collins effect within the same approach and at the same level of accuracy see [19]. SSA and Sivers effect in the Drell-Yan process have been discussed in [20].

In the following sections, we will first concentrate on unpolarized cross sections, applying Eq. (4) to several processes in different kinematical situations. Some details of the approach may differ for different processes and will therefore be discussed in the appropriate sections. We now briefly comment on how some more general ingredients required in practical calculations based on Eq. (4) have been fixed.

1) Concerning the $\boldsymbol{k}_{\perp}$-dependent PDF's (FF's) we assume that the $x(z)$ and $\boldsymbol{k}_{\perp}$ dependences factorize; we use a Gaussian-like, flavour-independent behaviour for the $\boldsymbol{k}_{\perp}$-dependent part; that is, neglecting for the moment the dependence on the factorization scale, we take $\left(k_{\perp}=\left|\boldsymbol{k}_{\perp}\right|\right)$

$$
\hat{f}_{a / A}\left(x, \boldsymbol{k}_{\perp a}\right)=f_{a / A}(x) \frac{\beta^{2}}{\pi} e^{-\beta^{2} k_{\perp a}^{2}} ; \quad \hat{D}_{C / c}\left(z, \boldsymbol{k}_{\perp C}\right)=D_{C / c}(z) \frac{\beta^{\prime 2}}{\pi} e^{-\beta^{\prime 2} k_{\perp C}^{2}}
$$

where the parameter $\beta\left(\beta^{\prime}\right)$ is related to the average partonic (hadronic) $k_{\perp}$ by the simple relation $1 / \beta\left(\beta^{\prime}\right)=$ $\left\langle k_{\perp a(C)}^{2}\right\rangle^{1 / 2}$.

2) The cross sections for the elementary, partonic scattering, $d \hat{\sigma}^{a b \rightarrow c d} / d \hat{t}$, are calculated at leading order in the strong coupling constant power expansion, including in the partonic kinematics the full dependence on the intrinsic transverse momenta $\boldsymbol{k}_{\perp}$. That is, the partonic cross sections in Eq. (4) will depend on the properly $\boldsymbol{k}_{\perp}$-modified partonic invariants $\hat{s}, \hat{t}$ and $\hat{u}$. At relatively low $p_{T}$ the inclusion of $\boldsymbol{k}_{\perp}$ dependence might make one or more of the partonic Mandelstam variables become smaller than a typical hadronic scale. This configuration would correspond to a situation where the propagator of the exchanged particle in the partonic scattering becomes soft; the observed transverse momentum is thus generated mainly by fluctuations in the intrinsic $\boldsymbol{k}_{\perp}$ distribution and not by the hard scattering. In this case perturbation theory would break down. In order to avoid such a problem and extend this approach down to $p_{T}$ around 1-2 GeV/c (where most of data on single spin asymmetries have been collected) different ways have been proposed. Following [2], we introduce a regulator mass, $\mu=0.8 \mathrm{GeV}$, shifting all partonic Mandelstam variables, that is we take

$$
\hat{t} \rightarrow \hat{t}-\mu^{2}, \quad \hat{u} \rightarrow \hat{u}-\mu^{2}, \quad \hat{s} \rightarrow \hat{s}+2 \mu^{2}
$$

3) Another somehow related source of potential ambiguity is the behaviour of the strong coupling constant, $\alpha_{s}\left(Q^{2}\right)$, in the low $Q^{2}$ regime. We adopt the prescription originally proposed by Shirkov and Solovtsov [21], using for $\alpha_{s}$ the expression

$$
\alpha_{s}\left(Q^{2}\right)=\frac{1}{\beta_{0}}\left[\frac{1}{\log \left(Q^{2} / \Lambda^{2}\right)}+\frac{\Lambda^{2}}{\Lambda^{2}-Q^{2}}\right]
$$

where as usual $\beta_{0}=\left(33-2 n_{f}\right) / 12 \pi, n_{f}$ being the number of active flavours (we use $\left.n_{f}=4\right)$, and $\Lambda=0.2 \mathrm{GeV} / c$. According to Eq. (9), at large $Q^{2} \alpha_{s}$ reduces to the standard LO expression, while at low $Q^{2}$ its behaviour is well under control without the introduction of any extra parameter, like a freezing scale parameter or a dynamical gluon mass. We have explicitly checked that other prescriptions give similar results.

Note that for the Drell-Yan process Eq. (4) simplifies a lot (see section II A); moreover, the large scale involved (namely the invariant mass of the observed lepton pair) removes the danger of critical kinematical regions discussed above.

4) Another important ingredient is the choice of the factorization scale $Q$ (we use one single scale for renormalization and factorization scales) governing the pQCD evolution of PDF's and FF's and entering the QCD running coupling constant, $\alpha_{s}$. It is well known that LO calculations, and in some cases NLO too, can strongly depend on this choice. Moreover, there is not in the literature a unique prescription for the choice of the factorization scale $Q$ in inclusive hadron production, and several possible alternatives have been suggested. Typical scales adopted are the observed hadron transverse momentum, $p_{T}$, or $p_{T} / 2$, and the transverse momentum of the fragmenting parton in the partonic c.m frame, $\hat{p}_{T}^{*}$, or $\hat{p}_{T}^{*} / 2$. Apart from the Drell-Yan process, where the natural scale is the invariant mass of the produced lepton pair, throughout this paper we will adopt the factorization scale $Q=\hat{p}_{T}^{*} / 2$.

5) Theoretical results for cross sections at LO and NLO are usually compared to get an estimate of the uncertainty related to the choice of the factorization scale and indications on the convergence of the perturbative expansion. The ratio of cross sections evaluated at NLO and LO respectively gives the so-called $K$-factor for the process under 
consideration. It is often assumed [2] that this ratio is independent of intrinsic transverse momentum effects, so that it can be estimated in the usual collinear pQCD approach. Throughout this paper we will conform to this prescription; that is, in comparing our LO results for cross sections with the corresponding experimental results, we will include collinear pQCD $K$-factors evaluated by means of independent numerical codes [22-24]. For the processes under consideration in this paper, the $K$-factors show some general behaviour with respect to the relevant kinematical variables [24]: they decrease smoothly with the increasing of the observed hadron transverse momentum, $p_{T}$ (the variation being steeper at the lower $p_{T}$ considered), at fixed $\sqrt{s}$; they also decrease mildly with $\sqrt{s}$ at fixed $p_{T}$, whereas they show a tiny dependence on $x_{F}$. For simplicity and clarity, in our plots of cross sections we will always show our numerical curves rescaled by a fixed (for that curve) $K$-factor, obeying the general trends of above and chosen according to the results of the numerical code INCNLL of Ref.s [22, 23]. We think this should help in clarifying the role played by $K$-factors in our results. The mild variations of the computed $K$-factor for each point of a given curve should not change our general conclusions. More details on the estimate of $K$-factors are given in the following sub-sections.

Let us finally recall that our main aim is not to perform an overall best fit to the available data (to this end, a full NLO approach would be required). Rather, we want to show to what extent the same approach adopted in the description of SSA is able, when complemented by collinear pQCD $K$-factors, to give a reasonably good account of a large set of cross-section data for different processes in different kinematical situations. We feel that the approach here described is reliable in this respect. Notice also that, as we have explicitly checked, the main interest of our study, namely single spin asymmetries, are not strongly affected by the choices discussed above.

In the following sub-sections we discuss separately and in more detail the processes under study.

\section{A. Drell-Yan process}

Let us now consider lepton pair production in $p p, p \bar{p}$ collisions, the Drell-Yan process. In LO, collinear pQCD the lepton pair has no transverse momentum, $\boldsymbol{q}_{T}$, in the hadronic center of mass frame. On the other hand plenty of data show a well-established $q_{T}$ spectrum, with an exponential behaviour at low $q_{T}(\lesssim 2-3 \mathrm{GeV} / c)$ turning into a power-like one at larger transverse momentum. NLO corrections and soft gluon radiation are important contributions both for the normalization of the cross section and in order to reproduce the observed power-like behaviour at large $q_{T}$. However, to describe the behaviour at very small $q_{T}$ one has to include the intrinsic parton momentum, $\boldsymbol{k}_{\perp}$.

Therefore, within our approach the Drell-Yan process can be considered a useful tool to gain information on the intrinsic transverse momentum of partons inside the initial colliding hadrons.

Analogously to Eq. (4), the cross section for the production of an $\ell^{+} \ell^{-}$pair in the collision of two hadrons $A$ and $B$ (there is no need for any fragmentation function here) reads:

$$
d \sigma=\sum_{a b} \int d x_{a} d^{2} \boldsymbol{k}_{\perp a} d x_{b} d^{2} \boldsymbol{k}_{\perp b} \hat{f}_{a / A}\left(x_{a}, \boldsymbol{k}_{\perp a}\right) \hat{f}_{b / B}\left(x_{b}, \boldsymbol{k}_{\perp b}\right) \frac{\hat{s}}{x_{a} x_{b} s} d \hat{\sigma}^{a b \rightarrow \ell^{+} \ell^{-}}
$$

where the elementary cross section $d \hat{\sigma}$ for the process $a\left(p_{a}\right) b\left(p_{b}\right) \rightarrow \ell^{+}\left(p_{+}\right) \ell^{-}\left(p_{-}\right)$is given by:

$$
d \hat{\sigma}=\frac{1}{2 \hat{s}} \frac{d^{3} p_{+}}{2 E_{+}} \frac{d^{3} p_{-}}{2 E_{-}} \frac{1}{(2 \pi)^{2}} \delta^{4}\left(p_{a}+p_{b}-p_{+}-p_{-}\right) \overline{\left|M_{a b \rightarrow \ell^{+} \ell^{-}}\right|^{2}} .
$$

The differential cross section $d \sigma$ depends on the variables

$$
\hat{s} \equiv M^{2}=\left(p_{a}+p_{b}\right)^{2} \equiv q^{2}, \quad y=\frac{1}{2} \ln \frac{q_{0}+q_{L}}{q_{0}-q_{L}}, \quad \boldsymbol{q}_{T},
$$

that is the squared invariant mass, the rapidity and the transverse momentum of the lepton pair; $q_{0}, \boldsymbol{q}_{T}$ and $q_{L}$ are respectively the energy, transverse and longitudinal components, in the $A-B$ c.m. frame, of the four-vector $q=p_{a}+p_{b}=p_{+}+p_{-}$. Using the relations:

$$
\frac{d^{3} p_{-}}{2 E_{-}}=d^{4} p_{-} \delta\left(p_{-}^{2}\right) \quad p_{-}=q-p_{+} \quad d M^{2} d y=2 d q_{0} d q_{L}
$$

Eq. (10) can be written as

$$
\frac{d^{4} \sigma}{d y d M^{2} d^{2} \boldsymbol{q}_{T}}=\sum_{a b} \int d x_{a} d^{2} \boldsymbol{k}_{\perp a} d x_{b} d^{2} \boldsymbol{k}_{\perp b} \hat{f}_{a / A}\left(x_{a}, \boldsymbol{k}_{\perp a}\right) \hat{f}_{b / B}\left(x_{b}, \boldsymbol{k}_{\perp b}\right) \delta^{4}\left(p_{a}+p_{b}-q\right) \frac{\hat{s}}{x_{a} x_{b} s} \hat{\sigma}_{0}^{a b},
$$


where $\hat{\sigma}_{0}^{a b}$ is the total cross section for the $a b \rightarrow \ell^{+} \ell^{-}$process:

$$
\hat{\sigma}_{0}^{a b}=\int \frac{d^{3} p_{+}}{2 E_{+}} \frac{1}{(2 \pi)^{2}} \frac{1}{2 M^{2}} \delta\left(\left(q-p_{+}\right)^{2}\right) \overline{\left|M_{a b \rightarrow \ell^{+} \ell^{-}}\left(p_{+}, q\right)\right|^{2}} .
$$

For the kinematical regimes of interest in this paper the dominating elementary contribution to the Drell-Yan process is the lowest order electromagnetic interaction, $q \bar{q} \rightarrow \gamma^{*} \rightarrow \ell^{+} \ell^{-}$, so that $a, b=q, \bar{q}$ with $q=u, \bar{u}, d, \bar{d}, s, \bar{s}$ and

$$
\hat{\sigma}_{0}^{q \bar{q}}=\frac{4 \pi \alpha^{2} e_{q}^{2}}{9 M^{2}} .
$$

The Dirac- $\delta$ function accounting for energy-momentum conservation in Eq. (14) contains the factors

$$
\begin{aligned}
& \frac{1}{2} \delta\left(E_{a}+E_{b}-q_{0}\right) \delta\left(p_{z a}+p_{z b}-q_{L}\right)= \\
& \frac{1}{2} \delta\left(\left(x_{a}+x_{b}\right) \frac{\sqrt{s}}{2}+\left[\frac{k_{\perp a}^{2}}{x_{a} s}+\frac{k_{\perp b}^{2}}{x_{b} s}\right] \frac{\sqrt{s}}{2}-q_{0}\right) \times \\
& \delta\left(\left(x_{a}-x_{b}\right) \frac{\sqrt{s}}{2}-\left[\frac{k_{\perp a}^{2}}{x_{a} s}-\frac{k_{\perp b}^{2}}{x_{b} s}\right] \frac{\sqrt{s}}{2}-q_{L}\right) .
\end{aligned}
$$

In the following we shall only consider kinematical regions such that:

$$
q_{T}^{2} \ll M^{2} \quad k_{\perp a, b}^{2} \simeq q_{T}^{2},
$$

where Eq. (17) simplifies into the usual collinear condition:

$$
\frac{1}{2} \delta\left(E_{a}+E_{b}-q_{0}\right) \delta\left(p_{z a}+p_{z b}-q_{L}\right)=\frac{1}{s} \delta\left(x_{a}-\frac{M}{\sqrt{s}} e^{y}\right) \delta\left(x_{b}-\frac{M}{\sqrt{s}} e^{-y}\right) .
$$

The Gaussian shape adopted for the $\boldsymbol{k}_{\perp}$ dependent PDFs', Eq. (7), together with Eq. (19), allow us to analytically perform the integrations in Eq. (14) which becomes

$$
\frac{d^{4} \sigma}{d y d M^{2} d^{2} \boldsymbol{q}_{T}}=\frac{\hat{\sigma}_{0}}{\pi s} \frac{\beta^{2} \bar{\beta}^{2}}{\beta^{2}+\bar{\beta}^{2}} \exp \left[-\frac{\beta^{2} \bar{\beta}^{2}}{\beta^{2}+\bar{\beta}^{2}} q_{T}^{2}\right] \sum_{q} e_{q}^{2} f_{q / p}\left(x_{a}\right) f_{\bar{q} / p}\left(x_{b}\right),
$$

where $\beta, \bar{\beta}$ refer to the quark(antiquark) PDF respectively. By direct comparison with data we can then extract the $\beta, \bar{\beta}$ parameters. The numerical values obtained in this way are of course related to the set of $x$ dependent PDF's adopted; throughout this paper we use the MRST01 set [25]. In principle $\beta$ and $\bar{\beta}$ could be $x$ and flavour dependent, but in first approximation a reasonable description of data can be obtained by neglecting these dependences. In this case, $\bar{\beta}=\beta$ and $1 / \beta^{2}=\left\langle q_{T}^{2}\right\rangle / 2$.

There is a clear experimental evidence that the average transverse momentum $\left\langle q_{T}\right\rangle$ of the lepton pair increases with the c.m. energy, in agreement with pQCD calculations [26]. In our LO approach, see Eq. (20), this behaviour can be obtained by using an effective value of $\beta$ decreasing with c.m. energy. This way, one should more correctly interpret the parameter $\beta$ as representing the effects of the primordial transverse motion of partons plus a component coming from NLO corrections. Since our first aim here is to extract information on the primordial intrinsic momentum, we write

$$
\left\langle q_{T}^{2}\right\rangle=\left\langle q_{T}^{2}\right\rangle_{\text {intr }}+\left\langle q_{T}^{2}\right\rangle_{\text {pert }},
$$

where $\left\langle q_{T}^{2}\right\rangle_{\text {pert }} \propto \alpha_{s} s$, is a perturbative, energy dependent contribution, which will be estimated by comparison with data. A good description of data available in different kinematical regions can be obtained by choosing at the lowest c.m. energies considered, $\sqrt{s} \simeq 20 \mathrm{GeV}$,

$$
\left(\left\langle q_{T}^{2}\right\rangle_{\text {intr }} / 2\right)^{1 / 2}=1 / \beta_{0}=0.8 \mathrm{GeV} / c .
$$

In Fig.s 1-5 we compare our estimates of the Drell-Yan invariant cross section in $p p$ collisions, averaged over bins of the invariant mass $M$ and at fixed rapidity, as a function of $q_{T}$, with a collection of data from several experiments [27-29].

All curves are rescaled by proper $K$-factors varying in the range 1.5-1.8 in the different cases (see legend and caption of figures), in agreement with NLO calculations. The factorization scale is set to the lepton pair invariant mass.

One can see that experimental data are described very well for $q_{T}$ values up to 2-3 GeV/c, where (see Fig. 3) a power-like behaviour, entirely due to radiative effects, starts to set in. 


\section{B. Direct photon production}

Let us now consider inclusive prompt photon production in $p p$ collisions (for a compilation of data and a complete and detailed NLO analysis in collinear pQCD of prompt photon production in hadron-hadron interactions see [30]).

As pointed out in many papers (see for instance [31]) intrinsic transverse momentum effects can help in solving the discrepancy between experimental data and LO as well as NLO collinear pQCD calculations. In particular, what emerges [32] is that the steep $p_{T}$ dependence of the measured differential cross section cannot be explained by any new improved PDF's (mostly for gluons); on the contrary the introduction of intrinsic transverse momentum effects significantly improves the comparison between theoretical calculations and experimental data. An attempt to give a more firm theoretical foundation, in terms of Sudakov form factors, of the phenomenological Gaussian $\boldsymbol{k}_{\perp}$ smearing has also been carried out [33].

We employ here our LO pQCD approach (including proper $K$-factors from NLO collinear pQCD), neglecting possible photon fragmentation contributions, an issue related also to isolated photon cross sections measured at extreme high-energy collider experiments (not considered in our analysis) [30].

Starting again from Eq. (4), the invariant differential cross section for the process $p p \rightarrow \gamma X$ then reads

$$
E_{\gamma} \frac{d^{3} \sigma}{d^{3} \boldsymbol{p}_{\gamma}}=\sum_{a b} \int d x_{a} d^{2} \boldsymbol{k}_{\perp a} d x_{b} d^{2} \boldsymbol{k}_{\perp b} \hat{f}_{a / p}\left(x_{a}, \boldsymbol{k}_{\perp a}\right) \hat{f}_{b / p}\left(x_{b}, \boldsymbol{k}_{\perp b}\right) \frac{\hat{s}}{x_{a} x_{b} s} \frac{d \hat{\sigma}^{a b \rightarrow \gamma d}}{d \hat{t}} \frac{\hat{s}}{\pi} \delta(\hat{s}+\hat{t}+\hat{u})
$$

where the basic partonic processes are the Compton process $g q(\bar{q}) \rightarrow \gamma q(\bar{q})$ and the annihilation process $q \bar{q} \rightarrow \gamma g$.

By exploiting the elastic constraint, $\hat{s}+\hat{t}+\hat{u}=0$, one of the integrations can be easily carried out, which fixes one of the light-cone momentum fractions (e.g. $x_{b}$ ); the 5-dimensional integral left is handled with the help of a VEGAS Monte Carlo routine which properly takes into account the full kinematics and all required kinematical cuts (see also the Appendix).

The kinematical regime we are interested in is mainly the moderately large region of $p_{T}$ values $(1-4 \mathrm{GeV} / c)$, where a uniform $\boldsymbol{k}_{\perp}$ smearing on a steep falling $p_{T}$ distribution produces a significant enhancing factor.

Similar studies (and conclusions) can be found in $[3,4]$ which however consider only photon production in the central rapidity region. Here we extend this approach to larger $x_{F}$ values, in view of its interest in studying SSA. A semi-analytical analysis with estimates of the enhancing factor resulting from the inclusion of intrinsic transverse momenta, in the full range of $x_{F}$ values, is under completion and will be published elsewhere [34].

Our main results are compared in Fig.s 6, 7 and 8 with a representative set of experimental data for $p p$ collisions, both from fixed target experiments, E704 (FNAL) [35] and WA70 (CERN) [36], and from collider experiments, R806 (ISR) [37]. For fixed target experiments and c.m. energies below $40 \mathrm{GeV}$ we have adopted the same value of $\beta_{0}$ as extracted in the Drell-Yan process, $\beta_{0}=1.25(\mathrm{GeV} / c)^{-1}$, corresponding to $\left\langle k_{\perp}^{2}\right\rangle^{1 / 2}=1 / \beta_{0}=0.8 \mathrm{GeV} / c$. For larger energies (ISR), Fig. 8, a slightly smaller value of $\beta, \beta=1(\mathrm{GeV} / c)^{-1}$ seems to better reproduce the low $p_{T}$ distribution. As said, we adopt the factorization scale $Q=p_{T}^{*} / 2$, where $p_{T}^{*}$ is the photon transverse momentum in the partonic c.m. frame. Notice that the non-collinear parton configuration in the hadronic c.m. frame implies $p_{T}^{*} \neq p_{T}$.

We use a NLO $K$-factor which decreases mildly with $p_{T}$ at fixed energy $\left(K=1.6\right.$ for $1 \mathrm{GeV} / c<p_{T}<3 \mathrm{GeV} / c$ and $K=1.3$ for $\left.p_{T}>4 \mathrm{GeV} / c\right)$. This is consistent with direct calculations we performed using the numerical code by Aurenche et al. [22] for NLO order direct photon production. As an example, in Fig. 8, where for the sake of simplicity we adopted a fixed value, $K=1.5$, use of a $p_{T}$ dependent $K$-factor would imply an increase of the low $p_{T}$ edge (where $K \simeq 1.9$ ) and at the same time a reduction of the large $p_{T}$ tail (where $K \simeq 1.2$ ) in our estimates, leading to a slightly better agreement with the data than shown.

For comparison in Fig.s 6, 7 and 8 we also show the corresponding LO, collinear pQCD results. As expected, at very large $p_{T}$ the intrinsic transverse momentum effects are negligible. In fact, $\boldsymbol{k}_{\perp}$ contributions behave like $k_{\perp} / p_{T}$; moreover, at large $p_{T}$ the spectrum is less steep than on the lower part, where even a small smearing produces a big effect.

As it is clear from Fig. 7, collinear pQCD calculations may already give an accurate description of WA70 data $(\sqrt{s}=23 \mathrm{GeV})$. On the other hand, results including $\boldsymbol{k}_{\perp}$ effects compare with data equally well, while E704 data [35], even if at a slightly lower energy $(\sqrt{s}=19.4 \mathrm{GeV})$ and at lower $p_{T}$, are heavily underestimated without $\boldsymbol{k}_{\perp}$ effects.

Once more we think that the agreement between our calculations and experimental data is good enough for our purposes.

\section{Inclusive pion production}

Large $p_{T}$ pion production in hadronic collisions is certainly the most interesting inclusive process from many points of view. In this case the discrepancies between collinear pQCD calculations and experimental data are even more 
significant. This is especially true at c.m. energies below $60 \mathrm{GeV}$, where theoretical calculations can underestimate experimental data by a factor 10 or larger, depending also on the choice of the factorization scale (see also [9]). Again, the inclusion of intrinsic motion of partons has been advocated in order to improve the agreement between theory and experiment.

Most part of previous work has been addressed to inclusive pion production in the central rapidity region $[2,38]$. On the other hand, as already mentioned, most of the interesting and puzzling experimental results on SSA are in the region of intermediate and large positive $x_{F}$ values; therefore a consistent study of unpolarized cross sections in this kinematical regime and within the same model is mandatory.

To this end, we again turn to Eq. (4). The elastic constraint allows us to carry out explicitly the integration over $z$, see the Appendix for details. Again, we employ a VEGAS Monte Carlo routine to perform the remaining 8-dimensional integral with proper kinematical cuts.

With respect to the previous cases considered (the Drell-Yan process and prompt photon production) a new transverse momentum dependent function enters through the fragmentation process. Notice also that the unpolarized FF's are presently known with much less accuracy than the nucleon PDF's. In particular, all available parameterizations (with the exception of the set by Kretzer, Leader and Christova (KLC) [39]) for the pion FF's are based on $e^{+} e^{-}$ data for charged pion production, which do not allow to explicitly separate between $\pi^{+}$and $\pi^{-}$case; this separation can only be made under further assumptions, which remain to be tested. The $z$ and $\boldsymbol{k}_{\perp}$ dependences in the FF are chosen according to Eq. (7).

Concerning the factorization scale, $Q$, as for the case of prompt photon production we use $Q=\hat{p}_{T}^{*} / 2$, where $\hat{p}_{T}^{*}$ is the transverse momentum of parton $c$ in the partonic c.m. frame, that is $\hat{p}_{T}^{*}=(\hat{u} \hat{t} / \hat{s})^{1 / 2}$ (notice that in prompt photon production the outgoing elementary particle at LO is the photon itself).

\section{Neutral pion production}

Let us start considering inclusive neutral pion production. Using the $\boldsymbol{k}_{\perp}$-dependent PDF's as inferred from the analysis of the Drell-Yan process and comparing our results with a large selection of cross-section data we can extract the (flavour independent) $\beta^{\prime}$ parameter entering the pion FF, see Eq. (7). In this sub-section we will indicatively use the set by Kniehl, Kramer, and Pötter (KKP) [40]. An optimized choice of $\beta^{\prime}$ seems to favour an explicit $z$ dependence, which somehow spoils the simple factorized form of Eq. (7),

$$
1 / \beta^{\prime}(z)=\left\langle k_{\perp \pi}^{2}(z)\right\rangle^{1 / 2}=0.7 z^{0.8}(1-z)^{0.15} \mathrm{GeV} / c \quad[\mathrm{KKP}] .
$$

Estimates of NLO corrections are taken into account by rescaling our results by proper $K$-factors. With our choice of the factorization scale a $K$-factor decreasing with energy and approaching unity at very large energies [24] is to be expected. This trend has been also checked by direct calculations performed with the help of the NLO numerical code for inclusive pion production by Aversa et al. [23].

In Fig. 9 we present our estimates of the invariant differential cross section for the process $p p \rightarrow \pi^{0} X$ at different energies vs. $p_{T}$ and at central rapidity, $y=0$. After rescaling each curve by the proper $K$-factor, ranging from 2.5 to 1, we get reasonable agreement with experimental data [41-44] over a large range of energies (from 20 to $200 \mathrm{GeV}$ ).

A bit more problematic is the case of neutral pion production in the very forward region (very small scattering angles), as shown in Figs. 10 and 11. In particular for $\theta<10^{0}-15^{0}$ even with the inclusion of intrinsic $\boldsymbol{k}_{\perp}$ effects the discrepancy between theory and data at ISR energies [41], $\sqrt{s}=23.3$ and $52.8 \mathrm{GeV}$, is still large. Nonetheless in these plots the LO collinear calculations (rescaled by the same $K$-factors) show how important the enhancing factor due to $\boldsymbol{k}_{\perp}$ contributions can be.

In Fig. 12 we compare our estimates, rescaled by a $K$-factor $K=2.5$, with data at $\sqrt{s}=19.4 \mathrm{GeV}$ [42]; here fair agreement can be reached over a broad region of $x_{F}$ away from 0 , and at different $p_{T}$ values. To our knowledge, these data are the only ones available covering almost the same kinematical region of the well-known E704 results on pion SSA [45].

Recent data from STAR [46] in comparable $x_{F}$ and $p_{T}$ ranges but at much larger energies are shown in Fig. 13, together with our theoretical curves, rescaled by proper $K$-factors. Let us remind that we use for each curve a fixed $K$-factor, whereas use of a $p_{T}$-dependent $K$-factor would imply an increase of the low $E_{\pi}$ edge and a reduction of the large $E_{\pi}$ tail. In this case we overestimate the data, but still a reasonable description is obtained. Let us notice that, since data on pion SSA come mainly from E704 experiment, in performing our optimized choice for $\beta^{\prime}$, we have given a slight preference to unpolarized cross-section data in the same kinematical region [42]. A smaller value of $1 / \beta^{\prime}$ would lead to a better agreement with STAR data without spoiling the good description of FNAL experimental results [42]. Let us also recall, in this respect, that the results presented cover a huge spectrum of different kinematical situations at different c.m. energies. 
We have also checked that the pion FF set by Kretzer [47] with a corresponding proper choice of the $\beta^{\prime}$ parameter,

$$
1 / \beta^{\prime}(z)=\left\langle k_{\perp \pi}^{2}(z)\right\rangle^{1 / 2}=1.4 z^{1.2}(1-z)^{0.35} \mathrm{GeV} / c \quad \text { [Kretzer] }
$$

gives similar results. For the processes under consideration here the KLC set [39] is practically equivalent to the set of Ref. [47].

\section{Charged pion production}

The study of inclusive charged pion production needs some additional caution; as it was already mentioned the available pion $\mathrm{FF}$ sets come from fits to experimental data on hadron production in $e^{+} e^{-}$annihilation, where only the sum of charged pions is detected. In order to get flavour (or charge) separation one therefore has to rely on some extra assumptions: for instance Kretzer [47] imposes, besides isospin symmetry, the following behaviour of the non-leading quark FF's at the input scale $\left(\mu_{0}^{2}\right)$ :

$$
\begin{aligned}
& D_{\pi^{+} / d}\left(z, \mu_{0}^{2}\right)=(1-z) D_{\pi^{+} / u}\left(z, \mu_{0}^{2}\right), \\
& D_{\pi^{+} / s}\left(z, \mu_{0}^{2}\right)=D_{\pi^{+} / d}\left(z, \mu_{0}^{2}\right) .
\end{aligned}
$$

Under proper pQCD evolution, a FF set for $\pi^{0}$ and $\pi^{ \pm}$can then be extracted. In other parameterizations, like the KKP set [40], no additional flavour separation assumption is made and only a FF set for neutral pions is available.

Clearly the relative weight between valence (or leading) and sea (or non-leading) quark FF's can play a relevant role in determining the relative yields of $\pi^{+}$and $\pi^{-}$.

For these reasons, we model two charged pion FF sets, starting from the neutral pion KKP parameterization. In one case (KKP-1) we assume that the non-leading (sea) quark FF's into a charged pion are all equal to the strange quark FF into a neutral pion: this leads to a strongly valence-dominated fragmentation mechanism at large $z$ values. A second case (KKP-2) is defined along the lines of Kretzer set. More precisely, assuming isospin symmetry, $D_{\pi^{-} / u}=D_{\pi^{+} / d}$ and charge conjugation invariance, $D_{\pi^{-} / u}=D_{\pi^{+} / \bar{u}}$, we define for KKP-1

$$
D_{\pi^{+} / d}(z)=D_{\pi^{0} / s}(z),
$$

that implies

$$
D_{\pi^{+} / u}(z)=2 D_{\pi^{0} / u}(z)-D_{\pi^{0} / s}(z) \quad(\mathrm{KKP}-1) .
$$

For KKP-2 set instead we impose

$$
D_{\pi^{+} / d}(z)=(1-z)^{a} D_{\pi^{+} / u}(z)
$$

that implies

$$
D_{\pi^{+} / u}(z)=2 D_{\pi^{0} / u}(z) /\left[1+(1-z)^{a}\right] \quad \quad(\mathrm{KKP}-2),
$$

where the parameter $a$, chosen reasonably around 1-1.5, will be fixed to $a=1.3$ in the following. Clearly, these sets leave unchanged, and equal to those of the KKP set, the FF's for neutral pions.

Let us remark that the analysis of Ref. [39], based on a simultaneous fit to $e^{+} e^{-}$and semi-inclusive DIS results which in principle allows flavour separation, indicates a relatively small suppression of non-leading vs. leading quark FF's, as already assumed in Kretzer set.

In Fig. 14 we show our estimates for the ratio of $\pi^{-}$and $\pi^{+}$yields and compare them with FNAL experimental data [48] for three different energies and at scattering angle $\theta=77 \mathrm{mrad}$ (at these energies, this corresponds almost to $x_{F}=0$ ) as a function of $p_{T}$. Results using the three different options for pion FF's discussed above (Kretzer, KKP-1 and KKP-2) are shown. Since all pion FF sets adopted assume that the FF for a neutral pion coincides with the average of those for charged pions, our estimates for $\pi^{0}$ cross sections, Fig.s 9-13, together with the results of Fig. 14, give complete information for the charged pion sector. Therefore, we do not explicitly show our results for charged pion cross sections, which are in good agreement with data.

From the ratio of $\pi^{-}, \pi^{+}$yields we see that both Kretzer and KKP-2 sets give a reasonably good description of the experimental data, while KKP-1 set seems to underestimate the data, in particular at large $p_{T}$. Let us however remark that the KKP-1 and KKP-2 sets are model sets based on some assumptions which remain to be tested. We propose them here as representative of two very different behaviours with respect to the relative weight of leading and non-leading pion FF's. As such, our results are not intended to give preference to any set of FF's over the others. 


\section{TRANSVERSE SINGLE SPIN ASYMMETRIES AND SIVERS EFFECT}

We come now to the other main subject of this paper. Parton intrinsic transverse momentum effects, as we have seen in the previous sections, can play a crucial role in reducing the discrepancies between pQCD estimates and experimental data for unpolarized cross sections in inclusive hadron production. What is more important from our point of view, they can be an essential ingredient in understanding some spin phenomena in the framework of pQCD. The large SSA observed in many reactions like $A^{\uparrow} B \rightarrow C X$ or $A B \rightarrow H^{\uparrow} X$ (where $H$ is typically a hyperon) can be described by certain spin-dependent effects generated by soft mechanisms in the presence of intrinsic transverse momentum. These effects and several applications to different processes, including various possible mechanisms of this sort, have been discussed in a series of papers [7, 10], keeping only dominant $\boldsymbol{k}_{\perp}$ contributions and under some simplifying assumptions about parton kinematics and $\boldsymbol{k}_{\perp}$ distributions.

Let us then consider the transverse single spin asymmetry

$$
A_{N}=\frac{d \sigma^{\uparrow}-d \sigma^{\downarrow}}{d \sigma^{\uparrow}+d \sigma^{\downarrow}}
$$

where $d \sigma^{\uparrow, \downarrow}$ stands for the invariant differential cross section for the process $A^{\uparrow, \downarrow}+B \rightarrow C+X$, and $\uparrow, \downarrow$ indicates the transverse direction with respect to the production plane (corresponding to the $\pm Y$ direction in a reference system where the initial polarized hadron moves along the $+Z$ direction and the observed hadron lies in the $+X-Z$ half-plane). As it was already mentioned, at leading twist there are three possible soft mechanisms (and three corresponding new spin and $\boldsymbol{k}_{\perp}$ dependent PDF's/FF's) contributing to this process, see Eq.s (5), (6), often referred to as "odd under naive time reversal":

a) The Sivers effect (and distribution function) [6], corresponding to the possible azimuthal dependence (around the light-cone direction of the parent nucleon) of the number density of unpolarized quarks (or gluons) inside a transversely polarized nucleon.

b) The Collins effect (and fragmentation function) [11], corresponding to the azimuthal dependence (around the light-cone direction of the fragmenting parton) of the number density of unpolarized hadrons resulting from the fragmentation of a transversely polarized quark (or linearly polarized gluon).

c) The Boer-Mulders effect (and distribution function) [14, 15], corresponding to the azimuthal dependence (around the light-cone direction of the parent nucleon) of the number density of transversely polarized quarks (linearly polarized gluons) inside an unpolarized nucleon.

When considering the production of a transversely polarized, spin-1/2 hadron (like e.g. in the process $A B \rightarrow H^{\uparrow} X$ ) another leading twist, $T$-odd effect has to been taken into account in the fragmentation process:

d) The so-called polarizing fragmentation function [17], corresponding to the azimuthal dependence (around the lightcone direction of the fragmenting parton) of the number density of transversely polarized hadrons resulting from the fragmentation of an unpolarized quark (or gluon).

It should be noted that in the pure parton model, where partons are treated as physical, massless free particles, all these effects, when described as totally independent, factorized processes, vanish [49].

Let us remind that these functions may be defined also for gluons; in this case, however, instead of transverse quark polarization we must refer to linear gluon polarization $[16,50]$.

All of the above spin effects a)-c) could contribute in principle to the process $p^{\uparrow} p \rightarrow \pi+X$, but, at the present stage, a complete phenomenological analysis is out of reach and perhaps not very significant, due to the relatively scarce experimental information available. On the other hand, it is very important to verify whether any of these possible contributions, when taken alone, may contribute and to what extent to the SSA observed, extracting even gross features of the corresponding PDF's/FF's.

In the following we will then concentrate on SSA in inclusive pion and photon production generated by the Sivers effect [6]. SSA in Drell-Yan processes within the same approach have been considered in Ref. [20]. For a complementary phenomenological study along the same lines, but invoking only Collins effect, see Ref. [19].

A more formal and complete analysis including all possible leading-order, leading-twist contributions for single and double spin asymmetries in inclusive pion production is currently under way and will be presented elsewhere [16].

Let us then start from the most general expression for the number density of unpolarized quarks $q$ (or analogously gluons), with light-cone momentum fraction $x$ and transverse momentum $\boldsymbol{k}_{\perp}$, inside a proton with transverse polarization $\boldsymbol{P}$ and three-momentum $\boldsymbol{p}$. One can write

$$
\hat{f}_{q / p^{\uparrow}}\left(x, \boldsymbol{k}_{\perp}\right)=\hat{f}_{q / p}\left(x, k_{\perp}\right)+\frac{1}{2} \Delta^{N} f_{q / p^{\uparrow}}\left(x, k_{\perp}\right) \hat{\boldsymbol{P}} \cdot\left(\hat{\boldsymbol{p}} \times \hat{\boldsymbol{k}}_{\perp}\right),
$$

which implies

$$
\hat{f}_{q / p}\left(x, k_{\perp}\right)=\frac{1}{2}\left[\hat{f}_{q / p^{\uparrow}}\left(x, \boldsymbol{k}_{\perp}\right)+\hat{f}_{q / p^{\downarrow}}\left(x, \boldsymbol{k}_{\perp}\right)\right],
$$




$$
\Delta^{N} f_{q / p^{\uparrow}}\left(x, \boldsymbol{k}_{\perp}\right)=\hat{f}_{q / p^{\uparrow}}\left(x, \boldsymbol{k}_{\perp}\right)-\hat{f}_{q / p^{\downarrow}}\left(x, \boldsymbol{k}_{\perp}\right)=\Delta^{N} f_{q / p^{\uparrow}}\left(x, k_{\perp}\right) \sin \left(\phi_{P}-\phi_{k_{\perp}}\right),
$$

where $\phi_{P}$ and $\phi_{k_{\perp}}$ are respectively the azimuthal angle of the proton (transverse) polarization vector and of $\boldsymbol{k}_{\perp}$. Eq. (34) gives the unintegrated, $k_{\perp}$-dependent unpolarized PDF, while Eq. (35) defines the Sivers PDF, $\Delta^{N} f_{q / p^{\uparrow}}\left(x, k_{\perp}\right)$. Another common notation for the Sivers function is $f_{1 T}^{\perp}\left(x, k_{\perp}\right)[14]$; it should be noted that the two definitions are not completely equivalent, the exact relation being $\Delta^{N} f_{q / p^{\uparrow}}\left(x, k_{\perp}\right)=\left(-2 k_{\perp} / M_{p}\right) f_{1 T}^{\perp}\left(x, k_{\perp}\right)$.

In order to perform numerical estimates and carry out a comparison with available experimental data we introduce a simple model for the Sivers function (see also Ref. [20] for more details), similar to what was previously done for the so-called polarizing fragmentation function in Ref. [17].

Analogously to the case of the unpolarized PDF's and FF's, Eq. (7), we consider a simple factorized form for the Sivers function:

$$
\Delta^{N} f_{q / p^{\uparrow}}\left(x, k_{\perp}\right)=\Delta^{N} f_{q / p^{\uparrow}}(x) h\left(k_{\perp}\right) .
$$

In order to satisfy the positivity bound (see Eq.s (34), (35))

$$
\left|\Delta^{N} f_{q / p^{\uparrow}}\left(x, k_{\perp}\right)\right| \leq 2 \hat{f}_{q / p}\left(x, k_{\perp}\right), \quad \forall x, k_{\perp},
$$

we put

$$
\begin{aligned}
\Delta^{N} f_{q / p^{\uparrow}}(x) & =2 \mathcal{N}_{q}(x) f_{q / p}(x) \\
h\left(k_{\perp}\right) & =\mathcal{H}\left(k_{\perp}\right) \frac{\beta^{2}}{\pi} e^{-\beta^{2} k_{\perp}^{2}}
\end{aligned}
$$

and we simply need to require

$$
\left|\mathcal{N}_{q}(x) \mathcal{H}\left(k_{\perp}\right)\right| \leq 1 \quad \forall x, k_{\perp}
$$

We actually impose $\mathcal{N}$ and $\mathcal{H}$ to be separately smaller than unity in magnitude, by choosing simple functional forms and dividing each of them by its maximum value:

$$
\begin{gathered}
\mathcal{N}_{q}(x)=N_{q} x^{a_{q}}(1-x)^{b_{q}} \frac{\left(a_{q}+b_{q}\right)^{\left(a_{q}+b_{q}\right)}}{a_{q}^{a_{q}} b_{q}^{b_{q}}}, \quad\left|N_{q}\right| \leq 1 \\
\mathcal{H}\left(k_{\perp}\right)=\sqrt{2 e\left(\alpha^{2}-\beta^{2}\right)} k_{\perp} \exp \left[-\left(\alpha^{2}-\beta^{2}\right) k_{\perp}^{2}\right], \quad \alpha>\beta .
\end{gathered}
$$

Notice that $\mathcal{H}\left(k_{\perp}\right)$ must vanish at least like $k_{\perp}$ for $k_{\perp} \rightarrow 0$. Eq.s (39) and (42) imply:

$$
\Delta^{N} f_{q / p^{\uparrow}}\left(x, k_{\perp}\right)=2 \mathcal{N}_{q}(x) f_{q / p}(x) \frac{\beta^{2}}{\pi} \sqrt{2 e\left(\alpha^{2}-\beta^{2}\right)} k_{\perp} e^{-\alpha^{2} k_{\perp}^{2}} .
$$

Since the positivity bound requires $\alpha>\beta$, one can write $\alpha^{2}=\beta^{2} / r$, where $r$ is a positive parameter smaller than one. We will keep this last parameter flavour and $x$-independent.

Let us stress again that in principle one can also define a gluon Sivers function, for which the same expressions would be valid. In order to reduce the number of free parameters and assuming in first approximation that transverse spin- $\boldsymbol{k}_{\perp}$ effects are valence-dominated (certainly a reasonable assumption for particles produced at large positive values of $x_{F}$ ), we will restrict here to consider valence quarks, neglecting (for what concerns the Sivers effect only) possible contributions from sea quarks and gluons. We have explicitly checked that, for the processes under study, even maximizing the corresponding Sivers functions these contributions could play a role only at low $x_{F}$, where $A_{N}$ is almost negligible (see also Ref. [51]).

Under these assumptions we are left with seven free parameters which completely fix the Sivers function as given in Eq. (43); six for $\mathcal{N}_{q}(x)$ in Eq. (41), with $q=u, d$, plus $r=\beta^{2} / \alpha^{2}$ in Eq. (42).

\section{A. Sivers effect in $p^{\uparrow} p \rightarrow \pi X$}

By comparing our calculations for SSA in pion production with available experimental data we now try to fix the set of parameters entering the Sivers function. 
According to Eq. (4), the numerator of the SSA, Eq. (32), in terms of Sivers effect alone reads

$$
\begin{aligned}
E_{\pi} \frac{d \sigma^{\uparrow}}{d^{3} \boldsymbol{p}_{\pi}}-E_{\pi} \frac{d \sigma^{\downarrow}}{d^{3} \boldsymbol{p}_{\pi}} & =\sum_{a, b, c, d} \int d x_{a} d^{2} \boldsymbol{k}_{\perp a} d x_{b} d^{2} \boldsymbol{k}_{\perp b} d z d^{3} \boldsymbol{k}_{\pi} \delta\left(\boldsymbol{k}_{\pi} \cdot \hat{\boldsymbol{p}}_{c}\right) \Delta^{N} f_{a / p^{\uparrow}}\left(x_{a}, \boldsymbol{k}_{\perp a} ; Q^{2}\right) \hat{f}_{b / p}\left(x_{b}, \boldsymbol{k}_{\perp b} ; Q^{2}\right) \\
& \times \frac{\hat{s}}{x_{a} x_{b} s} \frac{d \hat{\sigma}^{a b \rightarrow c d}}{d \hat{t}}\left(x_{a}, x_{b}, \hat{s}, \hat{t}, \hat{u}\right) \frac{\hat{s}}{\pi} \delta(\hat{s}+\hat{t}+\hat{u}) \frac{1}{z^{2}} J\left(z,\left|\boldsymbol{k}_{\pi}\right|\right) \hat{D}_{\pi / c}\left(z, \boldsymbol{k}_{\pi} ; Q^{2}\right) .
\end{aligned}
$$

The denominator of the SSA, $d \sigma^{\uparrow}+d \sigma^{\downarrow}$, is simply given by twice the unpolarized cross section, Eq. (4).

Let us remind that we consider the kinematics of the reaction in the c.m. of the colliding protons, where $\uparrow, \downarrow$ indicates the transverse direction with respect to the production plane (corresponding to the $\pm Y$ direction when the initial polarized proton moves along the $+Z$ direction and the observed pion lies in the $+X-Z$ half-plane). According to Eq. (35), in this frame $\phi_{P}=\pi / 2$, and

$$
\Delta^{N} f_{a / p^{\uparrow}}\left(x_{a}, \boldsymbol{k}_{\perp a}\right)=\Delta^{N} f_{a / p^{\uparrow}}\left(x_{a}, k_{\perp a}\right) \cos \left(\phi_{k_{\perp a}}\right) .
$$

As already discussed in section II C, energy-momentum conservation in Eq. (44) allows us to carry out e.g. the integration over $z$ (see also the Appendix) leaving us with an 8-dimensional integral. This makes unpractical and very time-consuming a complete best-fit procedure over all seven free parameters. Moreover, a complete analysis of this kind would probably be premature and not very significant at this stage. Therefore, we here adopt a less rigorous approach and try to reproduce the experimental data, extracting an optimized choice for the Sivers function parameters from a limited set of choices in the available parameter phase-space.

To this end, we consider the experimental data for pion SSA of the E704 Collaboration [45], at $\sqrt{s}=19.4 \mathrm{GeV}$. These data cover a large range of (positive) values for $x_{F}$, with $p_{T}$ in the range [0.7-2.0] GeV/c. In what follows, we will use for our calculations an averaged value of $p_{T}=1.5 \mathrm{GeV} / c$. Although this value might be slightly higher than the effective averaged $p_{T}$ of the data set, it is well indicative of the kinematic regime analysed.

We start by noticing what the E704 data show: at large positive $x_{F}$ the SSA, $A_{N}$, for charged pions is large and almost of the same magnitude, but opposite in sign for $\pi^{+}$and $\pi^{-}$(positive for $\pi^{+}$). In order to describe these results in our approach invoking the Sivers effect alone we need a positive (negative) up (down) Sivers function, $\Delta^{N} f_{q / p^{\uparrow}}\left(x, k_{\perp}\right)$. This means that the non-leading flavour contributions (e.g. $\left.d \rightarrow \pi^{+}\right)$) enter with opposite sign (all other terms in Eq. (44) giving positive contributions) with respect to the leading ones (e.g. $u \rightarrow \pi^{+}$) in each charged pion SSA asymmetry. In principle this might reduce the magnitude of $A_{N}$ and in some cases prevent a good description of data. This is particularly true for $\pi^{-}$production, where cancellations among different flavour contributions may be stronger. In this case, in fact, the dominance of the $d \rightarrow \pi^{-}$over the $u \rightarrow \pi^{-}$contribution in the fragmentation process can be weakened by the expected dominance (at large values of $x$ ) of the $u$ over the $d$ PDF's of the polarized proton.

Let us also remind that the available sets of pion FF's are essentially for the neutral pion case, coming from fits to data with no flavour/charge separation. Therefore we will fix the parameters entering the Sivers function by reproducing at best the neutral pion SSA data; only in a second step, we look at $A_{N}$ for charged pion production. This is slightly different from the procedure employed in former studies [7], where the used FF set were valence-like dominated (similar to our KKP-1 model set) and, adopting a simplified kinematics, a simultaneous best fit of charged and neutral pion SSA was performed.

Within these assumptions we get for the three different FF sets discussed in Section II C 2, the following optimal sets of parameters for the Sivers function

$$
\begin{array}{llll}
N_{u}=+0.40 & a_{u}=3.0 & b_{u}=0.6 & \\
N_{d}=-1.00 & a_{d}=3.0 & b_{d}=0.5 & \\
& & & \\
N_{u}=+0.40 & a_{u}=3.6 & b_{u}=0.6 & \\
N_{d}=-0.55 & a_{d}=3.0 & b_{d}=0.3 & \\
& & & \\
N_{u}=+0.40 & a_{u}=2.0 & b_{u}=0.3 \\
N_{d}=-0.90 & a_{d}=2.0 & b_{d}=0.2 & (\mathrm{KKP}-1),
\end{array}
$$

In all three cases the parameter $r$ entering the Gaussian $k_{\perp}$ dependence of the Sivers function is fixed at $r=0.7$ (see also Ref. [20]).

Note that we are employing the MRST01 set [25] for the unpolarized PDF's entering Eq. (43) instead of the GRV94 set [52], adopted for instance in Ref. [20]; as a consequence, the parameters for the Sivers function are slightly different. 
Corresponding estimates for SSA are shown in Fig.s 15, 16, and 17. We first notice that using the Kretzer FF set, where the non-leading quark $\mathrm{FF}$ is relevant over a large range of $z$ values, a simultaneous good description of neutral and charged pion SSA's seems to be more difficult (see Fig. 15). In the charged pion case, in fact, cancellations between leading and non-leading contributions in the numerator of $A_{N}$ are more effective. Even maximizing in size the $N_{d}$ parameter, see Eq. (46), but keeping the agreement with $\pi^{0}$ SSA data, we are not able to give a description of the $A_{N}$ data as good as for the other two sets. Apparently, the best agreement with data is obtained with the KKP-2 model set (see Fig. 17) where, without any need of maximizing the $N_{d}$ parameter, see Eq. (48), the concordance with neutral and charged pion SSA is quite good. Concerning the KKP-1 model set, where non-leading quark FF's are strongly suppressed, it also allows a reasonable description of data (see Fig. 16), being a factor of 2 far from saturating the positivity bound for both flavours, Eq. (47). Notice that in all cases the $a, b$ parameters cannot be changed very much if one wants to correctly reproduce the shape of the SSA. Therefore, the above conclusions can hardly be changed by modifying these parameters.

We have also considered a different functional form for $\mathcal{H}\left(k_{\perp}\right)$ in the $k_{\perp}$-dependent part of the Sivers function, Eq.s (39) and (42):

$$
\mathcal{H}^{\prime}\left(k_{\perp}\right)=\frac{2 k_{\perp} M_{0}}{k_{\perp}^{2}+M_{0}^{2}}
$$

where $M_{0}$ is a typical hadronic scale parameter. While still ensuring the positivity bound, Eq. (40), this form is less (power-like) suppressed at large $k_{\perp}$ values as compared with the Gaussian-like form of Eq. (42) adopted in our calculations. We have explicitly checked that taking $M_{0}=1 / \beta=0.8 \mathrm{GeV} / c$ (a natural choice that also minimizes the number of free parameters), and keeping the same sets of parameters as given in Eq.s (46), (47), and (48), we are still able to describe the E704 data for $A_{N}$, at the same level of accuracy as shown in the plots discussed above.

Another result of this analysis, which consistently treats the full $\boldsymbol{k}_{\perp}$ kinematics in the calculation of both the numerator and the denominator of $A_{N}$, is that all the main features of the Sivers effect and Sivers function found in previous papers [7] based on a simplified kinematics, are confirmed. On the contrary, the inclusion of full partonic kinematics seems to substantially reduce the role of the Collins mechanism in the $p^{\uparrow} p \rightarrow \pi X$ process (see [19])

As a next step, we may now use these findings to give estimates of $A_{N}$ for pion production at the much larger energies reached at the RHIC collider, namely $\sqrt{s}=200 \mathrm{GeV}$, but almost in the same $p_{T}$ region (so that the sofar unknown pQCD evolution of the Sivers function is not relevant). In this sense the following estimates can be considered as real predictions of our approach. Indeed they were obtained in a preliminary version of this study [53] before the new STAR data for neutral pion SSA [54] became available. Note however that in this analysis we have not considered the possible role of Sudakov suppression factors [55].

In Fig. 18 we show our results obtained using the three sets of parameters for the Sivers function discussed above and compare them with STAR experimental data [54]. Curves are calculated at fixed pseudo-rapidity $\eta=3.8$, so that $p_{T}$ and $x_{F}$ are correlated. To be safe with our pQCD approach, we consider only the region of $p_{T}>1-1.5$ $\mathrm{GeV} / c$; our curves are then cut at $p_{T}$ values around $1.25 \mathrm{GeV} / c$ (which implies $x_{F} \gtrsim 0.3$ ). This comparison indicates reasonable agreement with data, although error bars are still quite big at large $x_{F}$. We stress the fact that, as predicted theoretically, sizable pion SSA, with features similar to those found in the E704 kinematical range, are confirmed experimentally even at such large c.m. energies.

\section{B. Sivers effect in $p^{\uparrow} p \rightarrow \gamma X$}

Direct photon production in singly polarized $p p$ collisions is definitely of interest in this context, since it allows (unambiguous) access to the Sivers effect. In this case there is clearly no contamination from fragmentation processes (Collins effect); in the positive, moderately large $x_{F}$ region only the Sivers effect could be responsible for such an asymmetry in our approach. Therefore, the numerator of the SSA, Eq. (32), is given by an expression similar to that for the unpolarized cross section, Eq. (23), substituting the unpolarized PDF $\hat{f}_{a / p}\left(x_{a}, \boldsymbol{k}_{\perp a}\right)$ with the corresponding Sivers distribution $\Delta^{N} f_{a / p^{\uparrow}}\left(x_{a}, \boldsymbol{k}_{\perp a}\right)$; the denominator is simply given by twice the unpolarized cross section.

Unfortunately the few available data, at very small $x_{F}$, are compatible with zero within the (large) errors. Therefore a comparison with our predictions is somehow inconclusive. No test of our assumption of a valence-quark dominated Sivers function is possible either.

In Fig. 19 we show our predictions, utilizing the three different Sivers function parameterizations considered, for the kinematics of the E704 experiment, that is at $\sqrt{s}=19.4 \mathrm{GeV}$ and fixed $p_{T}=2.7 \mathrm{GeV} / c$, as a function of $x_{F}$. The calculated asymmetry is sizable at medium-large values of $x_{F}$, and could be measurable in principle. Experimental data at intermediate $x_{F}$ values would be of great help.

We also show our results for STAR kinematics in Fig. 20, namely at $\sqrt{s}=200 \mathrm{GeV}$ and fixed rapidity $y=3.8$ : once again sizable SSA are found which could be checked experimentally. 
We can then conclude that SSA in direct photon production could be an important tool in understanding the Sivers mechanism. The same is true for any process where only one at a time of all possible effects might be at work.

This is the case for instance of the single polarized Drell-Yan process, where by suitably integrating on the angular variables identifying the leptonic scattering plane only the Sivers effect would survive [20]; another key process could be heavy meson production in hadronic collisions, where the dominance of quark and gluon annihilation channels can enhance or even select out the Sivers effect [51].

\section{CONCLUSIONS}

In this paper we have studied the role of partonic intrinsic transverse momentum in inclusive particle production in (un)polarized high-energy hadronic reactions. Several papers in the framework of LO and NLO collinear pQCD have already been devoted to this subject in recent years. Our analysis is different in that it is mainly aimed at stressing the connection with recent approaches to the problem of the large single spin asymmetries measured in inclusive pion production at different c.m. energies at large positive $x_{F}$ and moderately large $p_{T}$. A lot of theoretical work has shown that LO pQCD with the inclusion of partonic intrinsic transverse momentum and a new class of leading twist, spin and $\boldsymbol{k}_{\perp}$-dependent PDF's and FF's is a promising approach to this problem. However, in those papers it was never shown in detail to what extent this approach is able to reproduce unpolarized cross sections for the same processes in similar kinematical conditions. Therefore, in the first part of this paper we have addressed this problem in a systematic way, with a full account of $\boldsymbol{k}_{\perp}$ effects both in the PDF's/FF's and in the elementary partonic process. We have applied our approach to the Drell-Yan process and to inclusive pion and direct photon production in hadronic collisions in several kinematical situations of interest, performing a detailed comparison with available experimental data. Our results show that, with few noticeable exceptions, our LO approach (when complemented with proper NLO $K$-factors) is in reasonable agreement with a large set of experimental data for unpolarized cross sections. Therefore, this gives support to the validity of the same approach in the study of single spin asymmetries.

In the second part of the paper, we have then applied our approach to the study of SSA in inclusive pion and direct photon production, with the inclusion of Sivers contribution alone (different contributions, like the Collins effect, in these or other processes have been or will be studied elsewhere). We have shown that the E704 experimental results on pion SSA can be reproduced with good accuracy by using physically reasonable parameterizations of the Sivers function for valence quark contributions (therefore, neglecting sea-quark and gluon Sivers functions in first approximation).

These same parameterizations lead to predictions in agreement with measurements performed by the STAR Collaboration at the RHIC-BNL accelerator at much higher c.m. energies and similar $x_{F}$ and $p_{T}$ ranges.

We have also shown that our analysis of SSA in terms of the Sivers effect and with a full treatment of $\boldsymbol{k}_{\perp}$ effects is in good qualitative agreement and confirms all main results and conclusions of former studies performed keeping only leading contributions in $\boldsymbol{k}_{\perp}$ and using a simplified partonic configuration.

However, some additional comments and some words of caution, regarding our results on unpolarized cross sections, are in order. The use of LO and NLO pQCD calculations (already in the collinear configuration) in the study of relatively low $p_{T}$ data faces a number of formal problems and leads to some model dependent choices regarding e.g. the factorization scale and the strong coupling constant behaviour. Moreover, from the numerical point of view, the lower the $p_{T}$ values considered, the more relevant are these model dependences.

Our results, which cover a range of $p_{T}$ values among $1-15 \mathrm{GeV} / c$ for the cross sections and of a few $\mathrm{GeV} / c$ at most for the SSA, have been obtained by performing a number of choices that we believe very reasonable and are largely adopted in the literature. Admittedly, much work remains to be done, in particular on the more formal aspects of the approach. We can however conclude with good confidence that there is not presently any strong argument or evidence against the applicability of our approach to SSA calculations coming from unpolarized cross section results, in particular for the kinematical situations of relevance for SSA. Within the uncertainties inherent the pQCD approach discussed above (hopefully less relevant for the SSA which are expressed as ratios of cross sections) there is good agreement among our results and experimental data. Concerning those few cases where our approach clearly fails to reproduce the results, we want to notice the following: 1) We have considered a very large set of data in different kinematical situations and from both fixed target and collider experiments; 2) Even NLO pQCD in collinear configuration faces several problems in fitting simultaneously the large collection of data considered here.

Let us finally add some words concerning transverse hyperon polarization in unpolarized hadronic collisions. It was shown that our approach is in principle able to reproduce most of the striking features of the experimental data available; the role of the so-called polarizing fragmentation function was emphasized. However, a combined analysis of SSA and unpolarized cross sections, like that performed here for the Drell-Yan process and inclusive pion, photon productions, is still lacking. This problem deserves a separate analysis which is under way and will be presented elsewhere. 


\section{Acknowledgments}

We are grateful to M. Anselmino and E. Leader for a critical reading of the manuscript and for useful comments. A special thank to W. Vogelsang who helped us in understanding the role of NLO corrections. We acknowledge partial support by M.I.U.R. (Ministero dell'Istruzione, dell'Università e della Ricerca) under Cofinanziamento P.R.I.N. 2003.

\section{APPENDIX A: FULL $k_{\perp}$ KINEMATICS}

We give here a detailed treatment of partonic kinematics with proper inclusion of transverse momentum effects, along the same lines of [56]. Let us consider the hadronic reaction $A B \rightarrow C X$ in the $A$ - $B$ center of mass frame with $A$ moving along the positive $Z$ axis and fix the scattering plane as the $X-Z$ plane. The 4-momenta of hadrons $A, B, C$ read

$$
p_{A}^{\mu}=\left(E_{A}, 0,0, P\right) \quad p_{B}^{\mu}=\left(E_{B}, 0,0,-P\right) \quad p_{C}^{\mu}=\left(E_{C}, p_{T}, 0, p_{L}\right),
$$

with $E_{A, B}=\sqrt{P^{2}+m_{A, B}^{2}}$ and $E_{C}=\sqrt{p_{T}^{2}+p_{L}^{2}+m_{C}^{2}}$. For equal-mass initial hadrons $\left(m_{A}=m_{B}=m\right)$, we have $E_{A}=E_{B}=\sqrt{s} / 2$, with $s=\left(p_{A}+p_{B}\right)^{2}$.

For massless partons $a, b$ inside hadrons $A, B$ we introduce light-cone momentum fractions $x_{a}=p_{a}^{+} / p_{A}^{+}, x_{b}=p_{b}^{-} / p_{B}^{-}$ and transverse momenta $\boldsymbol{k}_{\perp a}, \boldsymbol{k}_{\perp b}$. Their four-momenta then read

$$
\begin{aligned}
& p_{a}^{\mu}=x_{a} w \frac{\sqrt{s}}{2}\left(1+\frac{k_{\perp a}^{2}}{x_{a}^{2} w^{2} s}, \frac{2 k_{\perp a}}{x_{a} w \sqrt{s}} \cos \phi_{a}, \frac{2 k_{\perp a}}{x_{a} w \sqrt{s}} \sin \phi_{a}, 1-\frac{k_{\perp a}^{2}}{x_{a}^{2} w^{2} s}\right), \\
& p_{b}^{\mu}=x_{b} w \frac{\sqrt{s}}{2}\left(1+\frac{k_{\perp b}^{2}}{x_{b}^{2} w^{2} s}, \frac{2 k_{\perp b}}{x_{b} w \sqrt{s}} \cos \phi_{b}, \frac{2 k_{\perp b}}{x_{b} w \sqrt{s}} \sin \phi_{b},-1+\frac{k_{\perp b}^{2}}{x_{b}^{2} w^{2} s}\right),
\end{aligned}
$$

where the factor $w$, defined as $w=\left[1+\sqrt{1-4 m^{2} / s}\right] / 2$, can be safely taken equal to one for high-energy processes, as considered in this paper. In Eq. (A2) $k_{\perp a, b}=\left|\boldsymbol{k}_{\perp a, b}\right|$, and $\phi_{a, b}$ are the azimuthal angles of parton $a, b$ three-momenta in the hadronic c.m. frame.

The four-momentum of fragmenting parton $c$ is given in terms of the observed hadron momentum $p_{C}^{\mu}$, of the light-cone momentum fraction $z=p_{C}^{+} / p_{c}^{+}$and of the transverse momentum of hadron $C$ with respect to parton $c$ light-cone direction, $\boldsymbol{k}_{\perp C}$. In order to have all transverse momenta defined in the hadronic c.m. frame, we define the (two-dimensional) transverse momentum, $\boldsymbol{k}_{\perp C}$, as a genuine three-momentum:

$$
\boldsymbol{k}_{C}=k_{C}\left(\sin \theta_{k_{C}} \cos \phi_{k_{C}}, \sin \theta_{k_{C}} \sin \phi_{k_{C}}, \cos \theta_{k_{C}}\right),
$$

and impose the orthogonality condition $\boldsymbol{k}_{C} \cdot \boldsymbol{p}_{c}=0$, see below. Keeping hadron and parton masses into account, the parton four-momentum, $p_{c}^{\mu}=\left(E_{c}, \boldsymbol{p}_{c}\right)$, is then given as

$$
\begin{aligned}
\boldsymbol{p}_{c} & =\frac{\sqrt{E_{c}^{2}-m_{c}^{2}}}{\sqrt{\boldsymbol{p}_{C}^{2}-k_{C}^{2}}}\left(\boldsymbol{p}_{C}-\boldsymbol{k}_{C}\right)=\frac{\sqrt{E_{c}^{2}-m_{c}^{2}}}{\sqrt{\boldsymbol{p}_{C}^{2}-k_{C}^{2}}}\left(p_{T}-k_{C} \sin \theta_{k_{C}} \cos \phi_{k_{C}},-k_{C} \sin \theta_{k_{C}} \sin \phi_{k_{C}}, p_{L}-k_{C} \cos \theta_{k_{C}}\right),(\mathrm{A}) \\
E_{c} & =\frac{E_{C}+\sqrt{\boldsymbol{p}_{C}^{2}-k_{C}^{2}}}{2 z}\left[1+\frac{z_{c}^{2}}{\left(E_{C}+\sqrt{\boldsymbol{p}_{C}^{2}-k_{C}^{2}}\right)^{2}}\right]
\end{aligned}
$$

When, as it is the case in this paper, hadron $C$ is a pion and only light quark and gluon fragmentation is considered, hadron and parton masses can safely be neglected in the kinematics; eqs. (A4) and (A5) then simplify to

$$
\begin{aligned}
\boldsymbol{p}_{c} & =\frac{E_{c}}{\sqrt{E_{C}^{2}-k_{C}^{2}}}\left(p_{T}-k_{C} \sin \theta_{k_{C}} \cos \phi_{k_{C}},-k_{C} \sin \theta_{k_{C}} \sin \phi_{k_{C}}, p_{L}-k_{C} \cos \theta_{k_{C}}\right), \\
E_{c} & =\frac{E_{C}+\sqrt{E_{C}^{2}-k_{C}^{2}}}{2 z} .
\end{aligned}
$$

On the other hand for heavy hadrons (like $\Lambda$ hyperons) and/or heavy quarks (like charm quark fragmentation into $D$ mesons), mass corrections can be comparable with $\boldsymbol{k}_{\perp}$ effects. 
Another ingredient entering our basic factorization formula, Eq. (4), is the Jacobian factor $J\left(z,\left|\boldsymbol{k}_{C}\right|\right)$ connecting the parton $c$ to hadron $C$ invariant phase space, defined as

$$
\frac{d^{3} \boldsymbol{p}_{c}}{E_{c}}=\frac{1}{z^{2}} J\left(z,\left|\boldsymbol{k}_{C}\right|\right) \frac{d^{3} \boldsymbol{p}_{C}}{E_{C}},
$$

which for collinear and massless particles reduces simply to $J=1$. In the most general case, after some algebra, one gets

$$
J\left(z,\left|\boldsymbol{k}_{C}\right|\right)=\frac{\left(E_{C}+\sqrt{\boldsymbol{p}_{C}^{2}-k_{C}^{2}}\right)^{2}}{4\left(\boldsymbol{p}_{C}^{2}-k_{C}^{2}\right)}\left[1-\frac{z^{2} m_{c}^{2}}{\left(E_{C}+\sqrt{\boldsymbol{p}_{C}^{2}-k_{C}^{2}}\right)^{2}}\right]^{2} \Rightarrow \frac{\left(E_{C}+\sqrt{E_{C}^{2}-k_{C}^{2}}\right)^{2}}{4\left(E_{C}^{2}-k_{C}^{2}\right)},
$$

where the last expression holds for massless partons and hadrons.

The orthogonality condition $\boldsymbol{k}_{C} \cdot \boldsymbol{p}_{c}=0$ mentioned above is realized through the following relation

$$
d^{2} \boldsymbol{k}_{\perp C}=d^{3} \boldsymbol{k}_{C} \delta\left(\boldsymbol{k}_{C} \cdot \hat{\boldsymbol{p}}_{c}\right),
$$

where $\hat{\boldsymbol{p}}_{c}$ is the unit vector along the direction of motion of parton $c$. By using Eqs. (A3) and (A4), Eq. (A10) becomes

$$
\begin{aligned}
d^{2} \boldsymbol{k}_{\perp C} & =k_{C} d k_{C} d \theta_{k_{C}} d \phi_{k_{C}} \frac{\sqrt{\boldsymbol{p}_{C}^{2}-k_{C}^{2}}}{p_{T} \sin \phi_{k_{C}}^{0}}\left[\delta\left(\phi_{k_{C}}-\phi_{k_{C}}^{0}\right)+\delta\left(\phi_{k_{C}}-\left(2 \pi-\phi_{k_{C}}^{0}\right)\right)\right], \\
\cos \phi_{k_{C}}^{0} & =\frac{k_{C}-p_{L} \cos \theta_{k_{C}}}{p_{T} \sin \theta_{k_{C}}}, 0 \leq \phi_{k_{C}}^{0} \leq \pi
\end{aligned}
$$

In this way the integration over $\phi_{k_{C}}$ can be carried out directly (notice that there are two possible solutions to be considered).

With the expression of parton momenta given in Eqs. (A2) and (A6) one can calculate the partonic Mandelstam invariants; by exploiting the elastic, massless parton constraint $\hat{s}+\hat{t}+\hat{u}=0$, one can in turn fix the value of $z$ :

$$
\begin{aligned}
& \hat{s}=\left(p_{a}+p_{b}\right)^{2}=x_{a} x_{b} s\left[1-2 \frac{k_{\perp a} k_{\perp b}}{x_{a} x_{b} s} \cos \left(\phi_{a}-\phi_{b}\right)+\frac{k_{\perp a}^{2} k_{\perp b}^{2}}{x_{a}^{2} x_{b}^{2} s^{2}}\right], \\
& \hat{t}=\left(p_{a}-p_{c}\right)^{2}=\frac{T}{z} \\
& \hat{u}=\left(p_{b}-p_{c}\right)^{2}=\frac{U}{z}, \\
& \hat{s} \delta(\hat{s}+\hat{t}+\hat{u})=z \delta\left(z+\frac{T+U}{\hat{s}}\right),
\end{aligned}
$$

where the two functions $T$ and $U$, easily obtained from the explicit expressions of parton momenta, are independent of $z$. For heavy quarks, one can still fix $z$ from the elastic constraint but in this case the expressions for $\hat{t}$ and $\hat{u}$, in terms of $z$, are more involved.

We now discuss, limiting to the massless case, the constraints on the full phase space entering our factorized expression for (un)polarized cross sections. Besides the trivial bounds $0<x_{a, b}, z<1,0 \leq \phi_{a, b} \leq 2 \pi$ and $0 \leq \theta_{k_{C}} \leq \pi$, we require that, even including intrinsic transverse momentum effects, $a$ ) each parton keeps moving along the same direction as its parent hadron, $\boldsymbol{p}_{a(b)} \cdot \boldsymbol{P}_{A(B)}>0$, and $b$ ) the parton energy is not larger than the parent hadron energy, $E_{a(b)} \leq E_{A(B)}$. This implies the following bounds

$$
k_{\perp a(b)} / \sqrt{s}<\min \left[x_{a(b)}, \sqrt{x_{a(b)}\left(1-x_{a(b)}\right)}\right] .
$$

Analogously, for the fragmentation process $c \rightarrow C+X$ we require $\boldsymbol{p}_{c} \cdot \boldsymbol{P}_{C}>0$ and $E_{C} \leq E_{c}$ (both fulfilled by Eq. (A7), where we have consistently disregarded the solution $\left.E_{c}=\left[E_{C}-\sqrt{E_{C}^{2}-k_{C}^{2}}\right] /(2 z)\right)$. The last constraint implies the following bound on $k_{C}$, at fixed $z$

$$
k_{C} / E_{C} \leq 1(z \leq 1 / 2) ; \quad k_{C} / E_{C} \leq 2 \sqrt{z(1-z)}(z>1 / 2) .
$$

By requiring $\left|\cos \phi_{k_{C}}^{0}\right| \leq 1$, see Eq. (A12), we have a further constraint on $k_{C}$, at fixed $\theta_{k_{C}}$, namely

$$
p_{L} \cos \theta_{k_{C}}-p_{T} \sin \theta_{k_{C}} \leq k_{C} \leq p_{L} \cos \theta_{k_{C}}+p_{T} \sin \theta_{k_{C}} .
$$


A word of caution is needed for the partonic flux factor when incoming partons are not collinear. As shown in [57] the correct convolution formula connecting the partonic to the hadronic process is expressed in terms of partonic and hadronic tensors, that is in terms of scattering amplitudes squared rather than directly in terms of cross sections. The convolution then only involves parton-hadron light-cone energy ratios and no partonic flux, which would depend on the relative azimuthal angle between parton momenta, enters.

More precisely, for the reaction $A B \rightarrow c d$ one has

$$
\begin{aligned}
|M|_{A B \rightarrow c d}^{2} & =\int d x_{a} d^{2} \boldsymbol{k}_{\perp a} d x_{b} d^{2} \boldsymbol{k}_{\perp b} \hat{f}_{a / A}\left(x_{a}, \boldsymbol{k}_{\perp a}\right) \hat{f}_{b / B}\left(x_{b}, \boldsymbol{k}_{\perp b}\right)|M|_{a b \rightarrow c d}^{2} \frac{P_{A}^{+} P_{B}^{+}}{p_{a}^{+} p_{b}^{+}} \\
& =\int d x_{a} d^{2} \boldsymbol{k}_{\perp a} d x_{b} d^{2} \boldsymbol{k}_{\perp b} \hat{f}_{a / A}\left(x_{a}, \boldsymbol{k}_{\perp a}\right) \hat{f}_{b / B}\left(x_{b}, \boldsymbol{k}_{\perp b}\right)|M|_{a b \rightarrow c d}^{2} \frac{1}{x_{a} x_{b}} .
\end{aligned}
$$

In order to obtain the proper normalized $A B \rightarrow c d$ cross section one has to divide Eq. (A20) by the hadronic flux (2s). On the other hand since our convolution formula is given in terms of partonic cross sections, which are normalized to the partonic flux $2 \hat{s}$, to restore proper normalization we have to rescale them by the factor $\hat{s} /\left(x_{a} x_{b} s\right)$.

[1] R.D. Field and R.P. Feynman, Phys. Rev. D15, 2590 (1977); R.P. Feynman, R.D. Field and G.C. Fox, Phys. Rev. D18, 3320 (1978).

[2] X.-N. Wang, Phys. Rev. C61, 064910 (2000); Y. Zhang, G. Fai, G. Papp, G. Barnaföldi, and P. Lévai, Phys. Rev. C65, 034903 (2002).

[3] C.Y Wong, and H. Wang, Phys. Rev. C58, 376 (1998).

[4] L. Apanasevich, et al. (E706 Collab.), Phys. Rev. Lett. 81, 2642 (1998).

[5] G.L. Kane, J. Pumplin, and W. Repko, Phys. Rev. Lett. 25, 1689 (1978).

[6] D. Sivers, Phys. Rev. D41, 83 (1990); Phys. Rev. D43, 261 (1991).

[7] M. Anselmino, M. Boglione, and F. Murgia, Phys. Lett. B362, 164 (1995); M. Anselmino, and F. Murgia, Phys. Lett. B442, 470 (1998).

[8] S.J. Brodsky, D.S. Hwang, and I. Schmidt, Phys. Lett. B530, 99 (2002); Nucl. Phys. B642, 344 (2002); J.C. Collins, Phys. Lett. B536, 43 (2002).

[9] C. Bourrely and J. Soffer, e-Print Archive: hep-ph/0311110.

[10] M. Anselmino, M. Boglione, and F. Murgia, Phys. Rev. D60, 054027 (1999); M. Anselmino, M. Boglione, J. Hansson, and F. Murgia, Eur. Phys. J. C13, 519 (2000); M. Boglione, and E. Leader, Phys. Rev. D61, 114001 (2000).

[11] J.C. Collins, Nucl. Phys. B396, 16 (1993).

[12] J.C. Collins, D.E. Soper and G. Sterman, Nucl. Phys. B250, 199 (1985); J.C. Collins and D.E. Soper, Nucl. Phys. B193, 381 (1981).

[13] X. Ji, J. Ma, F. Yuan, e-Print Archive: hep-ph/0404183; hep-ph/0405085.

[14] P.J. Mulders, and R.D. Tangerman, Nucl. Phys. B461, 197 (1996), erratum ibid. B484, 538 (1997); D. Boer, and P.J. Mulders, Phys. Rev. D57, 5780 (1998).

[15] D. Boer, Phys. Rev. D60, 014012 (1999).

[16] M. Anselmino, M. Boglione, U. D’Alesio, E. Leader, S. Melis, and F. Murgia, in progress.

[17] M. Anselmino, D. Boer, U. D'Alesio, and F. Murgia, Phys. Rev. D63, 054029 (2001); Phys. Rev. D65, 114014 (2002).

[18] V. Barone, A. Drago, and P.G. Ratcliffe, Phys. Rep. 359, 1 (2002).

[19] M. Anselmino, M. Boglione, U. D’Alesio, E. Leader, and F. Murgia, in progress.

[20] M. Anselmino, U. D'Alesio, and F. Murgia, Phys. Rev. D67, 074010 (2003).

[21] D.V. Shirkov and I.L. Solovtsov, Phys. Rev. Lett. 79, 1209 (1997).

[22] P. Aurenche, R. Baier, M. Fontannaz, and D. Schiff, Nucl. Phys. B297, 661 (1988).

[23] F. Aversa, P. Chiappetta, M. Greco, and J.Ph. Guillet, Nucl. Phys. B327, 109 (1989).

[24] W. Vogelsang, private communication.

[25] A.D. Martin, R.G. Roberts, W.J. Stirling, and R.S. Thorne, Phys. Lett. B531, 216 (2002).

[26] G. Altarelli, G. Parisi and R. Pretonzio, Phys. Lett. B76, 351 (1978); 356 (1978); H. Fritzch and P. Minkowski, Phys. Lett. B73, 80 (1978).

[27] A.S. Ito, et al., Phys. Rev. D23, 604 (1981).

[28] G. Moreno, et al., Phys. Rev. D43, 2815 (1991).

[29] D. Antreasyan, et al. Phys. Rev. Lett. 48, 302 (1982).

[30] W. Vogelsang and M.R. Walley, J. Phys. G23, Suppl. 7A, A1 (1997).

[31] J.F. Owens, Rev. Mod. Phys. 59, 465 (1987).

[32] J. Huston, E. Kovacs, S. Kuhlmann, H.L. Lai, J.F. Owens, and W.K. Tung, Phys. Rev. D51, 6139 (1995).

[33] H.L. Lai and Hsiang-nan Li, Phys. Rev. D58, 114020 (1998).

[34] U. D'Alesio, S. Melis, and F. Murgia in preparation.

[35] D.L. Adams, et al. (E704 Collab.), Phys. Lett. B345, 569 (1995). 
[36] M. Bonesini, et al., Z. Phys. C38, 371 (1988).

[37] E. Anassontzis et al. (R806/807 Collab.), Z. Phys. C13, 277 (1982).

[38] L. Apanasevich, et al. (E706 Collab.), Phys. Rev. D68, 052001 (2003).

[39] S. Kretzer, E. Leader, and E. Christova, Eur. Phys. J. C22, 269 (2001).

[40] B.A. Kniehl, G. Kramer, and B. Pötter, Nucl. Phys. B582, 514 (2000).

[41] D.L. Owen et al., Phys. Rev. Lett. 45, 89 (1980).

[42] G. Donaldson, et al., Phys. Lett. B73, 375 (1978).

[43] S.S. Adler et al. (PHENIX Collab.), Phys. Rev. Lett. 91, 241803 (2003).

[44] D.L. Adams, et al. (E704 Collab.), Phys. Rev. D53, 4747 (1996).

[45] D.L. Adams, et al. (E704 Collab.), Phys. Lett. B345, 569 (1995); B261, 197 (1991); B264, 462 (1991).

[46] J. Adams, et al. (STAR Collab.), Phys. Rev. Lett. 92, 171801 (2004).

[47] S. Kretzer, Phys. Rev. D62, 054001 (2000).

[48] D. Antreasyan, et al., Phys. Rev. D19, 764 (1979).

[49] E. Leader, e-Print Archive: hep-ph/0405284, and private communication.

[50] P.J. Mulders and J. Rodrigues, Phys. Rev. D63, 094021 (2001).

[51] M. Anselmino, M. Boglione, U. D'Alesio, E. Leader, and F. Murgia, e-Print Archive: hep-ph/0407100.

[52] M. Gluck, E. Reya, and A. Vogt, Z. Phys. C67, 433 (1995).

[53] U. D'Alesio and F. Murgia, AIP Conf. Proc. 675, 469 (2003);

[54] J. Adams, et al. (STAR Collab.), Phys. Rev. Lett. 92, 171801 (2004).

[55] D. Boer, Nucl. Phys. B603, 195 (2001).

[56] A.P. Contogouris, R. Gaskell, and S. Papadopoulos, Phys. Rev. D17, 2314 (1978);

[57] R.N. Cahn, Phys. Rev. D40, 3107 (1989). 


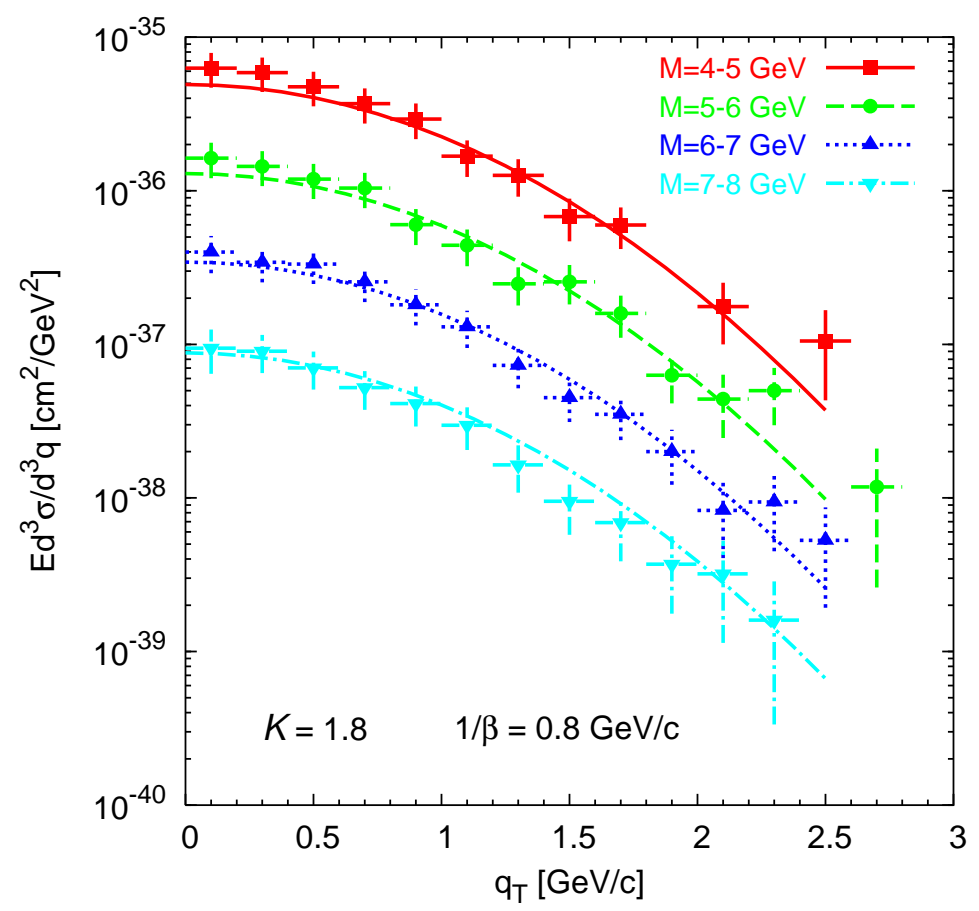

FIG. 1: Invariant differential cross section for the Drell-Yan process at $\sqrt{s} \simeq 19.4 \mathrm{GeV}$ and fixed rapidity $y=0.4$, as a function of the transverse momentum of the lepton pair $q_{T}$ and averaged over different invariant mass bins (see the legend). The parameterization MRST01 [25] for the unpolarized parton distributions is used, with $1 / \beta=0.8 \mathrm{GeV} / c$. Curves are rescaled by a fixed $K$-factor, $K=1.8$. Data are from [27].

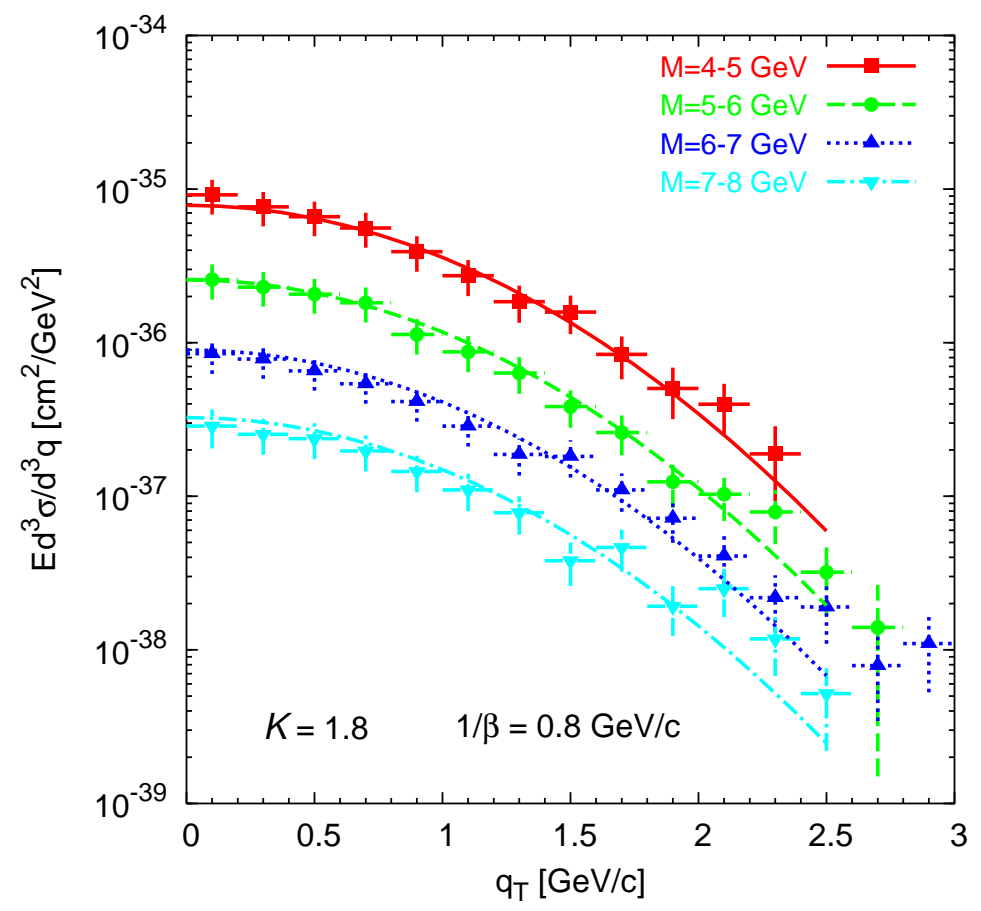

FIG. 2: Invariant differential cross section for the Drell-Yan process at $\sqrt{s} \simeq 23.8 \mathrm{GeV}$ and fixed rapidity $y=0.21$, as a function of the transverse momentum of the lepton pair $q_{T}$ and averaged over different invariant mass bins (see the legend). The parameterization MRST01 [25] for the unpolarized parton distributions is used, with $1 / \beta=0.8 \mathrm{GeV} / c$. Curves are rescaled by a fixed $K$-factor, $K=1.8$. Data are from [27]. 


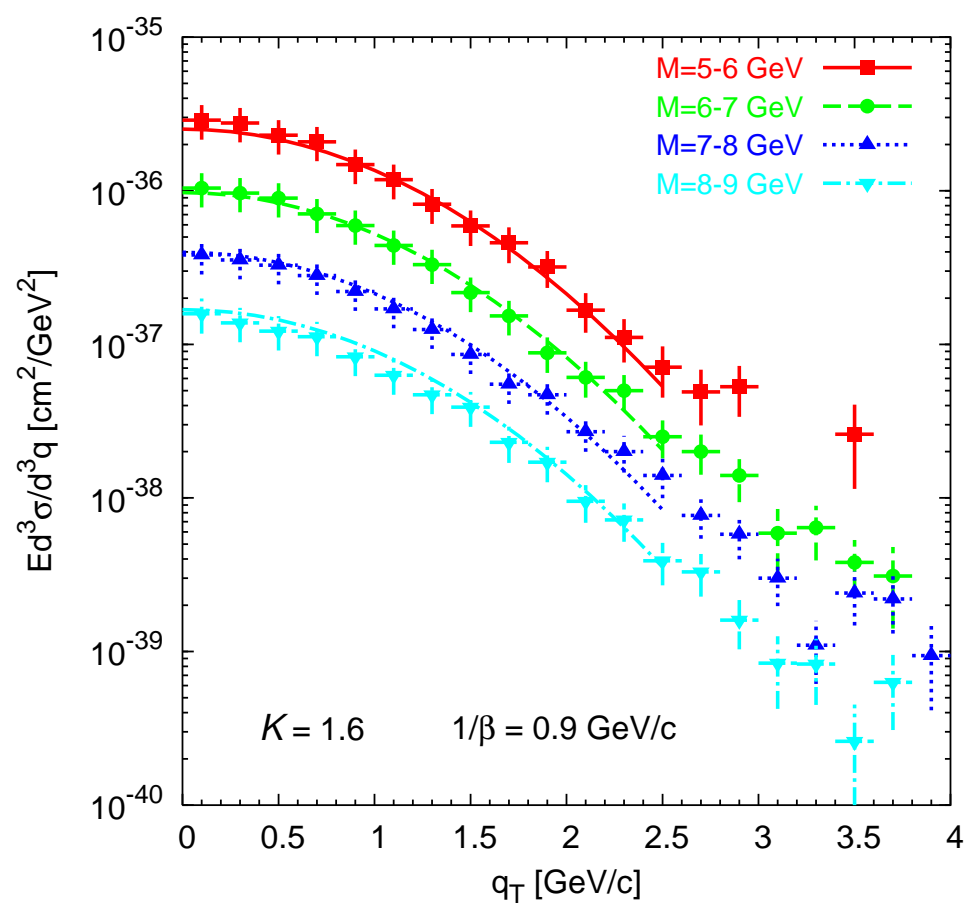

FIG. 3: Invariant differential cross section for the Drell-Yan process at $\sqrt{s} \simeq 27.4 \mathrm{GeV}$ and fixed rapidity $y=0.03$, as a function of the transverse momentum of the lepton pair $q_{T}$ and averaged over different invariant mass bins (see the legend). The parameterization MRST01 [25] for the unpolarized parton distributions is used, with $1 / \beta=0.9 \mathrm{GeV} / c$. Curves are rescaled by a fixed $K$-factor, $K=1.6$. Data are from [27].

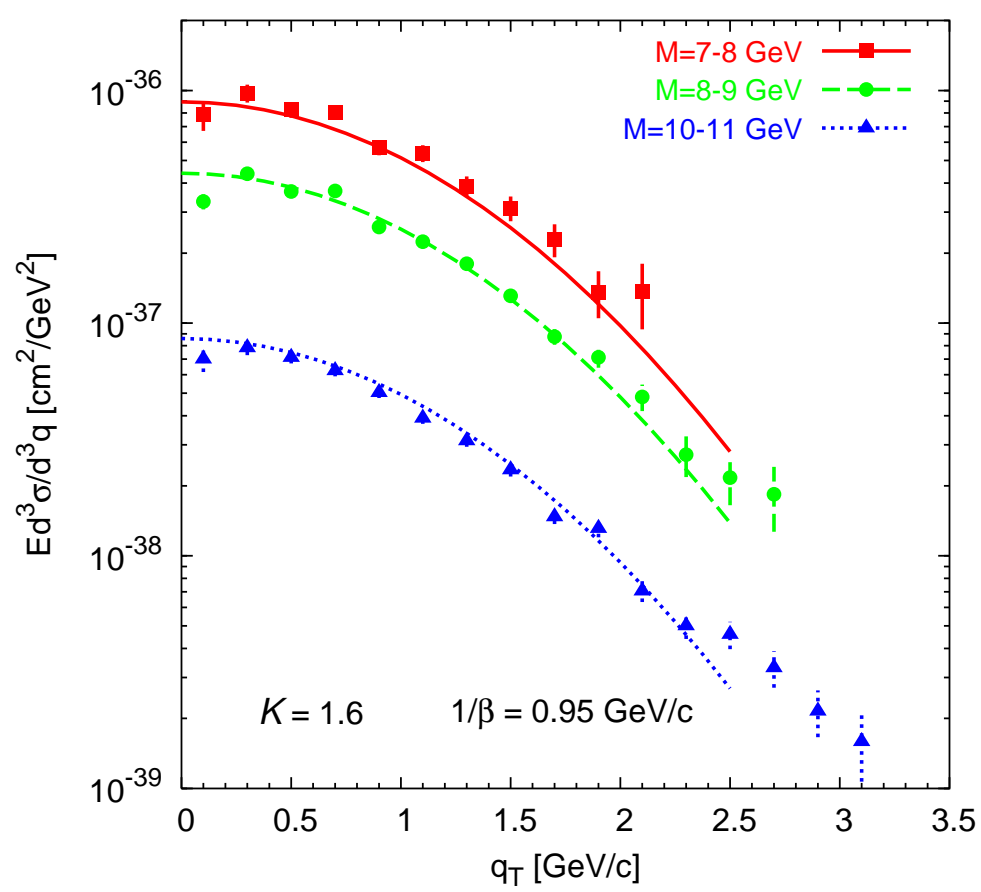

FIG. 4: Invariant differential cross section for the Drell-Yan process at $\sqrt{s} \simeq 38.8 \mathrm{GeV}$ and fixed $x_{F}=0.1$, as a function of the transverse momentum of the lepton pair $q_{T}$ and averaged over different invariant mass bins (see the legend). The parameterization MRST01 [25] for the unpolarized parton distributions is used, with $1 / \beta=0.95 \mathrm{GeV} / c$. Curves are rescaled by a fixed $K$-factor, $K=1.6$. Data are from [28]. 


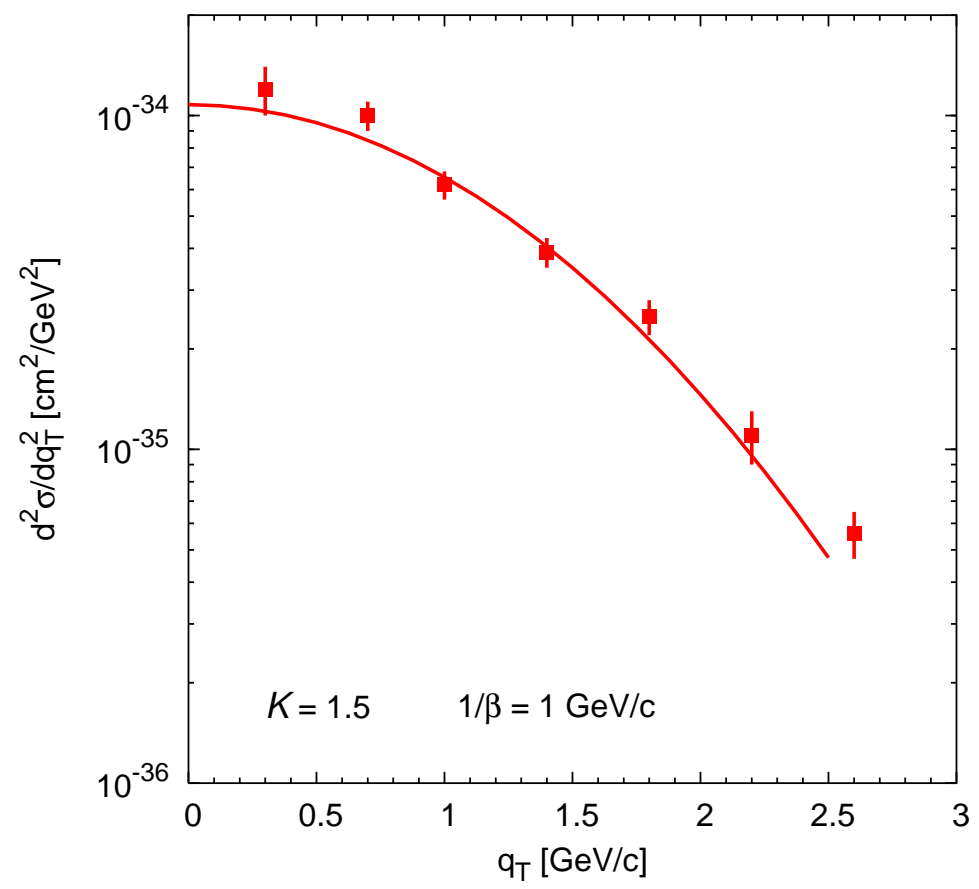

FIG. 5: Differential cross section for the Drell-Yan process at $\sqrt{s}=62 \mathrm{GeV}$, as a function of the transverse momentum of the lepton pair $q_{T}$ and averaged over the invariant mass bin $5 \mathrm{GeV}<M<8 \mathrm{GeV}$ and over the Feynman variable bin $-0.1<x_{F}<0.8,\left(x_{F}=2 q_{L} / \sqrt{s}\right)$. The parameterization MRST01 [25] for the unpolarized parton distributions is used, with $1 / \beta=1.0 \mathrm{GeV} / c$. The theoretical curve is rescaled by a fixed $K$-factor, $K=1.5$. Data are from [29].

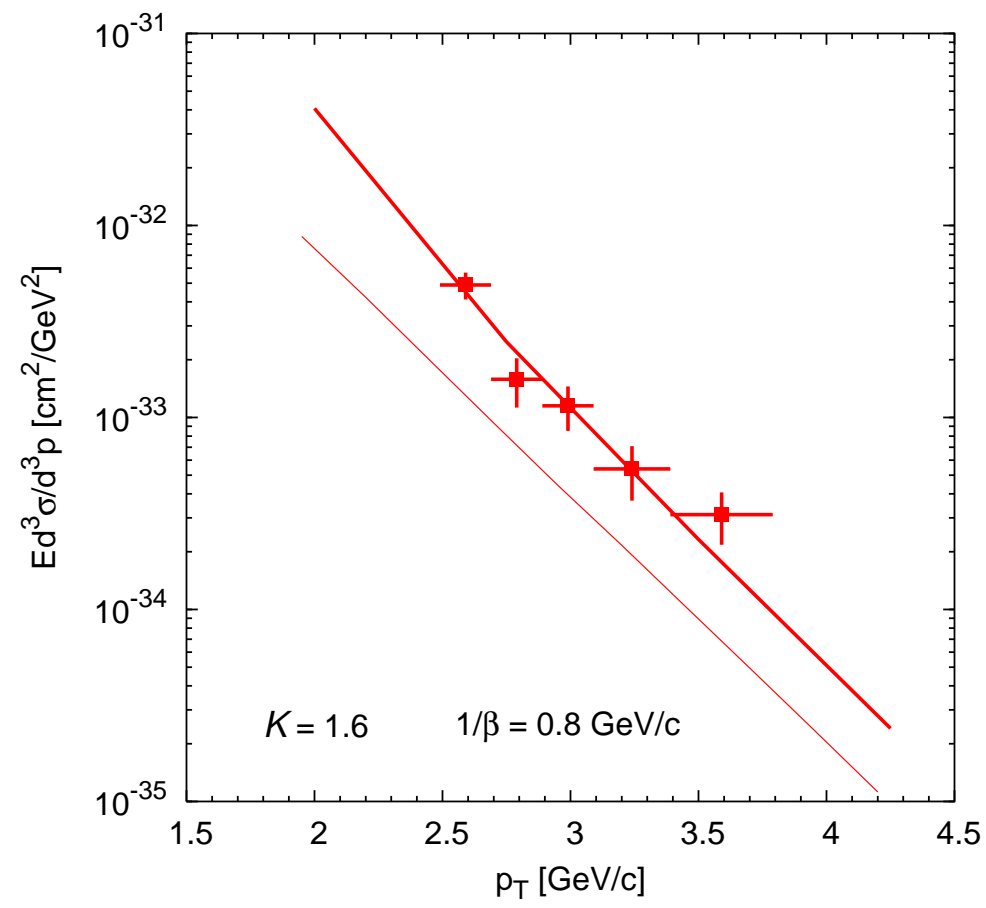

FIG. 6: Invariant differential cross section for prompt photon production in $p p$ collisions, at $\sqrt{s} \simeq 19.4 \mathrm{GeV}$ and fixed $x_{F}=0$, as a function of the photon transverse momentum $p_{T}$. The parameterization MRST01 [25] for the unpolarized parton distributions is used, with $1 / \beta=0.8 \mathrm{GeV} / c$ (thick line). For comparison, the result in collinear partonic configuration (thin line) is also shown. Both curves are rescaled by a fixed $K$-factor, $K=1.6$. Data [35] are averaged over the $x_{F}$ bin $-0.15<x_{F}<0.15$. 


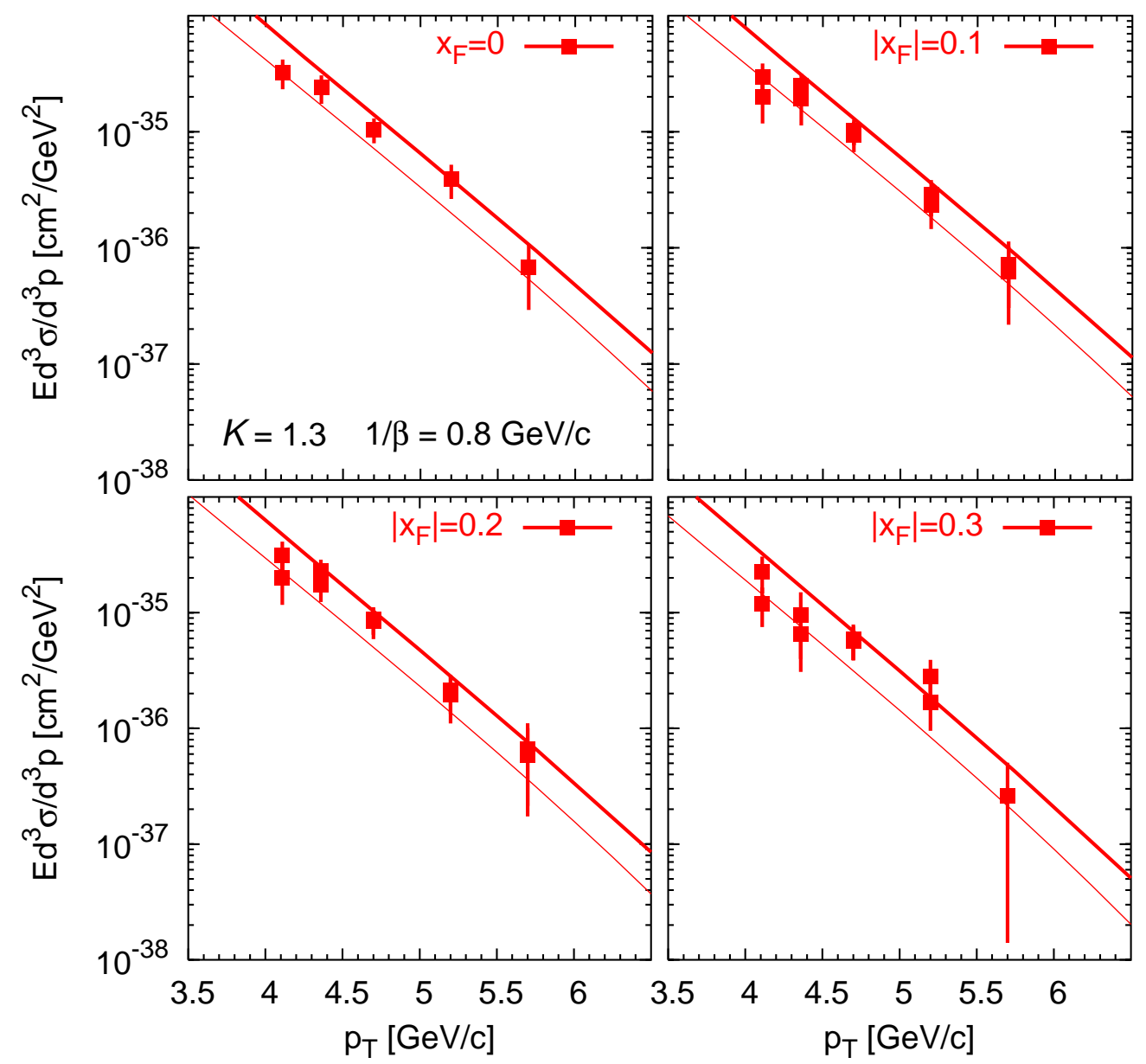

FIG. 7: Invariant differential cross section for prompt photon production in $p p$ collisions, at $\sqrt{s} \simeq 23 \mathrm{GeV}$ and different $x_{F}$ values, as a function of the photon transverse momentum $p_{T}$. The parameterization MRST01 [25] for the unpolarized parton distributions is used, with $1 / \beta=0.8 \mathrm{GeV} / c$ (thick lines). For comparison, the results in collinear partonic configuration (thin lines) are also shown. All curves are rescaled by a fixed $K$-factor, $K=1.3$. Data are from [36]. 


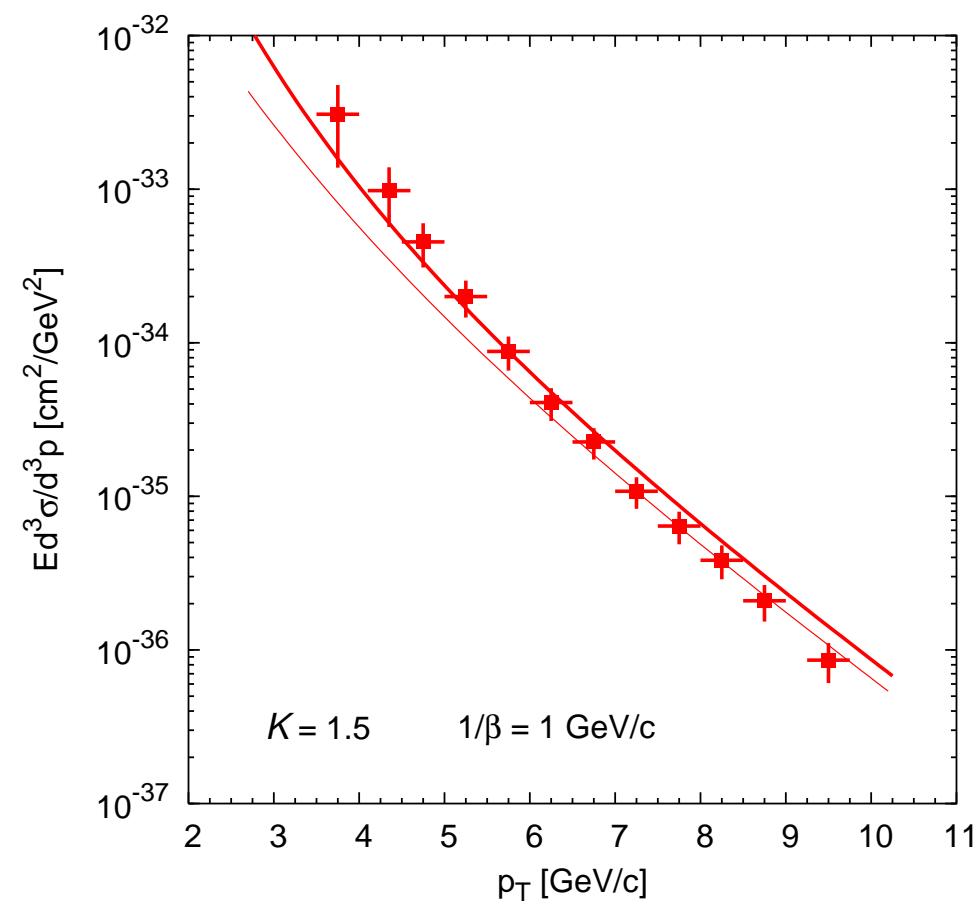

FIG. 8: Invariant differential cross section for prompt photon production in $p p$ collisions, at $\sqrt{s}=63$ GeV and fixed rapidity $y=0$, as a function of the photon transverse momentum $p_{T}$. The parameterization MRST01 [25] for the unpolarized parton distributions is used, with $1 / \beta=1.0 \mathrm{GeV} / c$ (thick line). For comparison, the result in collinear partonic configuration (thin line) is also shown. Both curves are rescaled by a fixed $K$-factor, $K=1.5$. Data [37] are averaged over the rapidity bin $-0.2<y<0.2$.

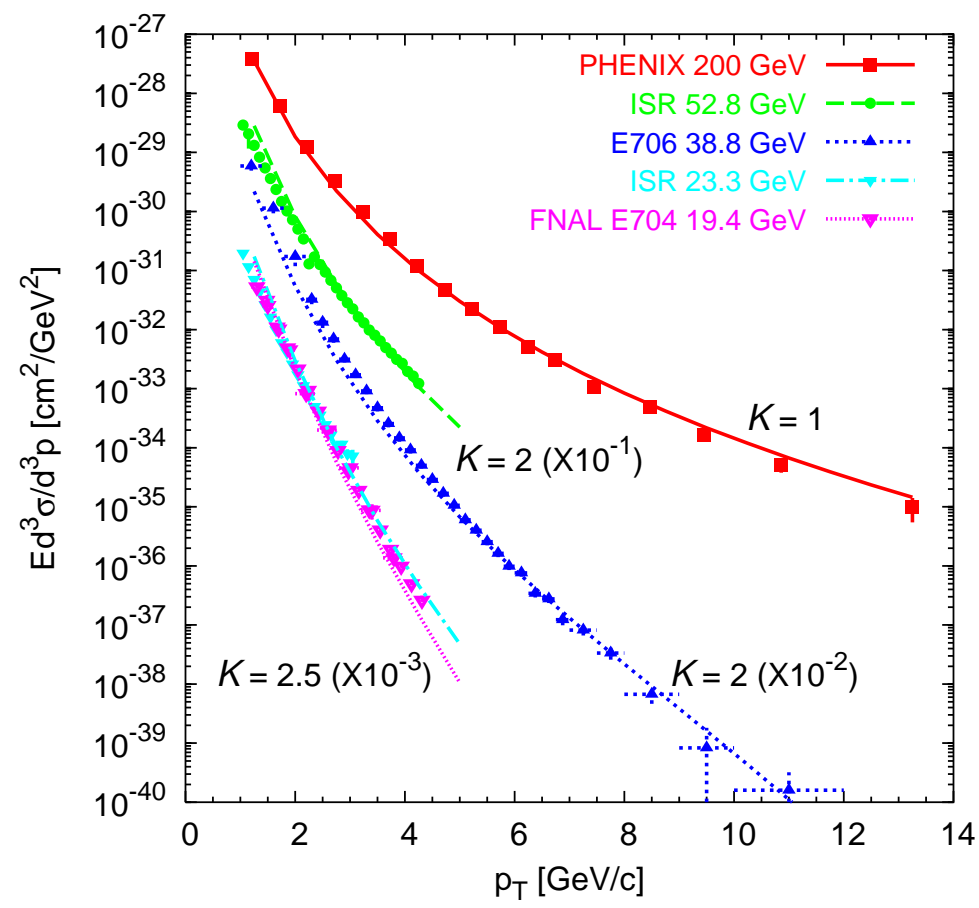

FIG. 9: Invariant differential cross section for inclusive $\pi^{0}$ production in $p p$ collisions, at different c.m. energies (see the legend) and fixed rapidity $y=0$, as a function of the pion transverse momentum $p_{T}$. We use the parameterization MRST01 [25] for the unpolarized parton distributions, with $1 / \beta=0.8 \mathrm{GeV} / c$, and the parameterization $\mathrm{KKP}$ [40] for the unpolarized fragmentation functions, with $\beta^{\prime}$ given in Eq. (24). $K$-factors for each case are shown (some data sets and their corresponding curves are further rescaled for clarity). Data are from [38, 41-44]. 


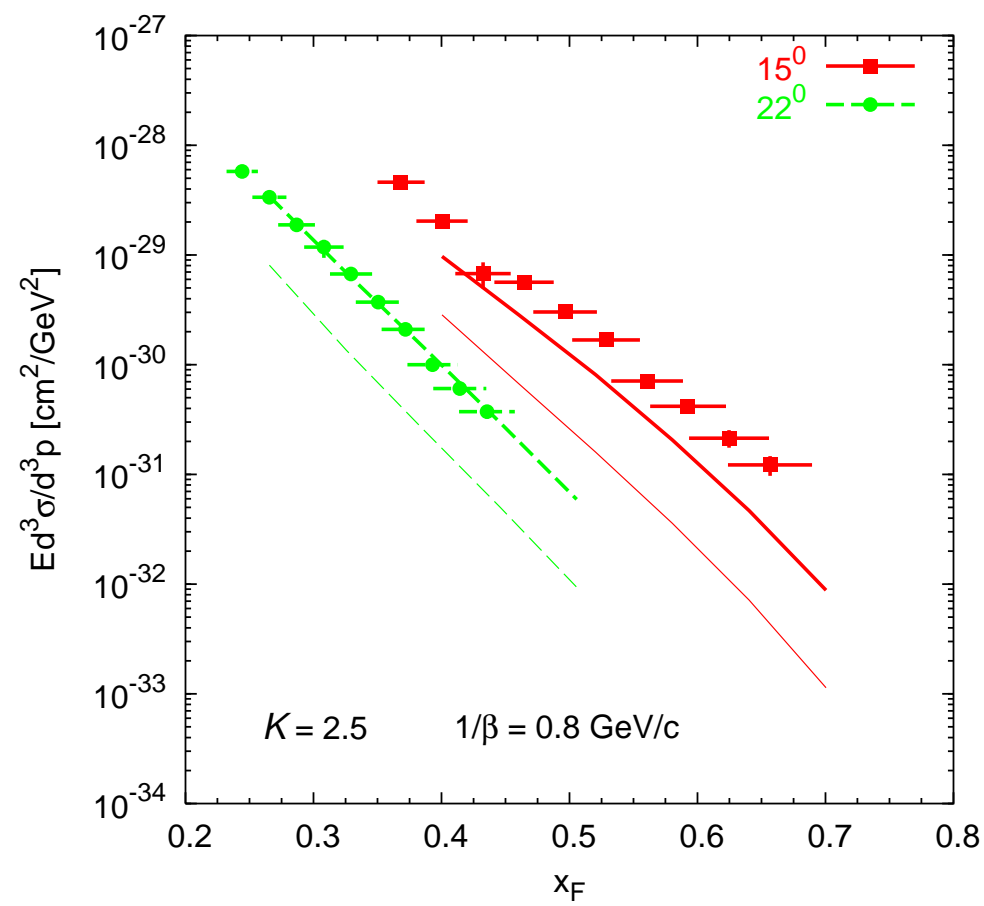

FIG. 10: Invariant differential cross section for inclusive $\pi^{0}$ production in $p p$ collisions, at $\sqrt{s}=23.3 \mathrm{GeV}$ and for two c.m. scattering angles, as a function of $x_{F}$. We use the parameterization MRST01 [25] for the unpolarized parton distributions, with $1 / \beta=0.8 \mathrm{GeV} / c$, and the parameterization KKP [40] for the unpolarized fragmentation functions, with $\beta^{\prime}$ given in Eq. (24) (thick lines). For comparison, the results in collinear partonic configuration (thin lines) are also shown. All curves are rescaled by a fixed $K$-factor, $K=2.5$. Data are from [41].

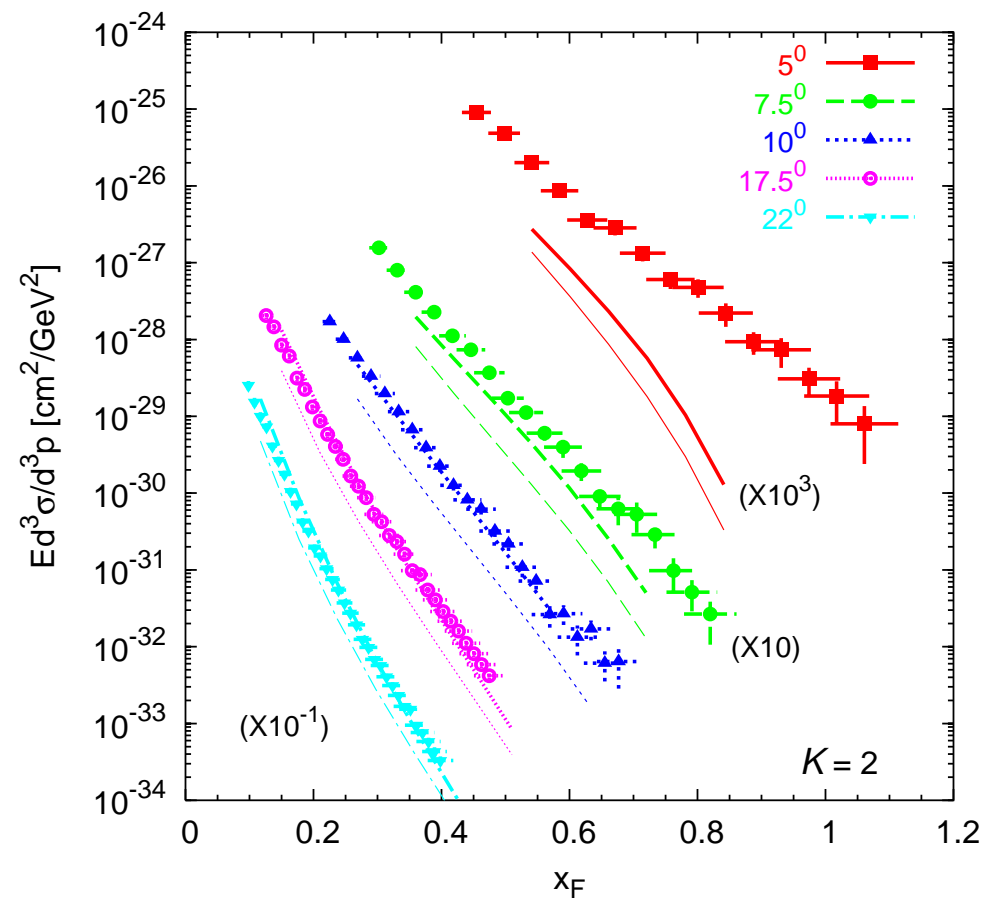

FIG. 11: Invariant differential cross section for inclusive $\pi^{0}$ production in $p p$ collisions, at $\sqrt{s}=52.8 \mathrm{GeV}$ and for different c.m. scattering angles (see the legend), as a function of $x_{F}$. We use the parameterization MRST01 [25] for the unpolarized parton distributions, with $1 / \beta=0.8 \mathrm{GeV} / c$, and the parameterization KKP [40] for the unpolarized fragmentation functions, with $\beta^{\prime}$ given in Eq. (24) (thick lines). For comparison, the results in collinear partonic configuration (thin lines) are also shown. All curves are rescaled by a fixed $K$-factor, $K=2$ (some data sets with their corresponding curves are further rescaled for clarity). Data are from [41]. 


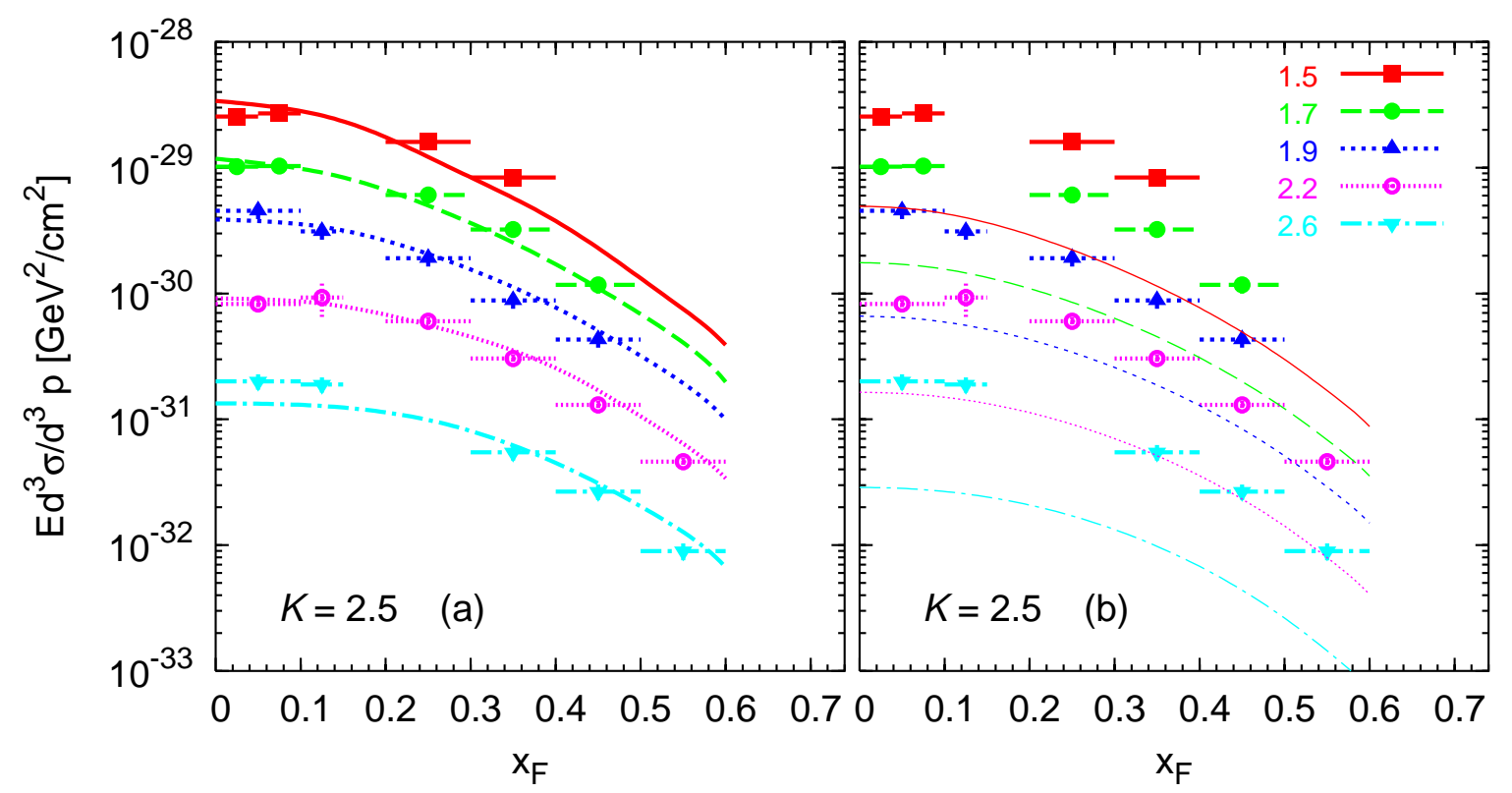

FIG. 12: Invariant differential cross section for inclusive $\pi^{0}$ production in $p p$ collisions, at $\sqrt{s}=19.4 \mathrm{GeV}$ and for different $p_{T}$ values (in $\mathrm{GeV} / c$, see the legend), as a function of $x_{F}$. We use the parameterization MRST01 [25] for the unpolarized parton distributions, with $1 / \beta=0.8 \mathrm{GeV} / c$, and the parameterization KKP [40] for the unpolarized fragmentation functions, with $\beta^{\prime}$ given in Eq. (24) (plot (a), thick lines). For comparison, the corresponding results in collinear partonic configuration (plot (b), thin lines) are also shown. All curves are rescaled by a fixed $K$-factor, $K=2.5$. Data are from [42].

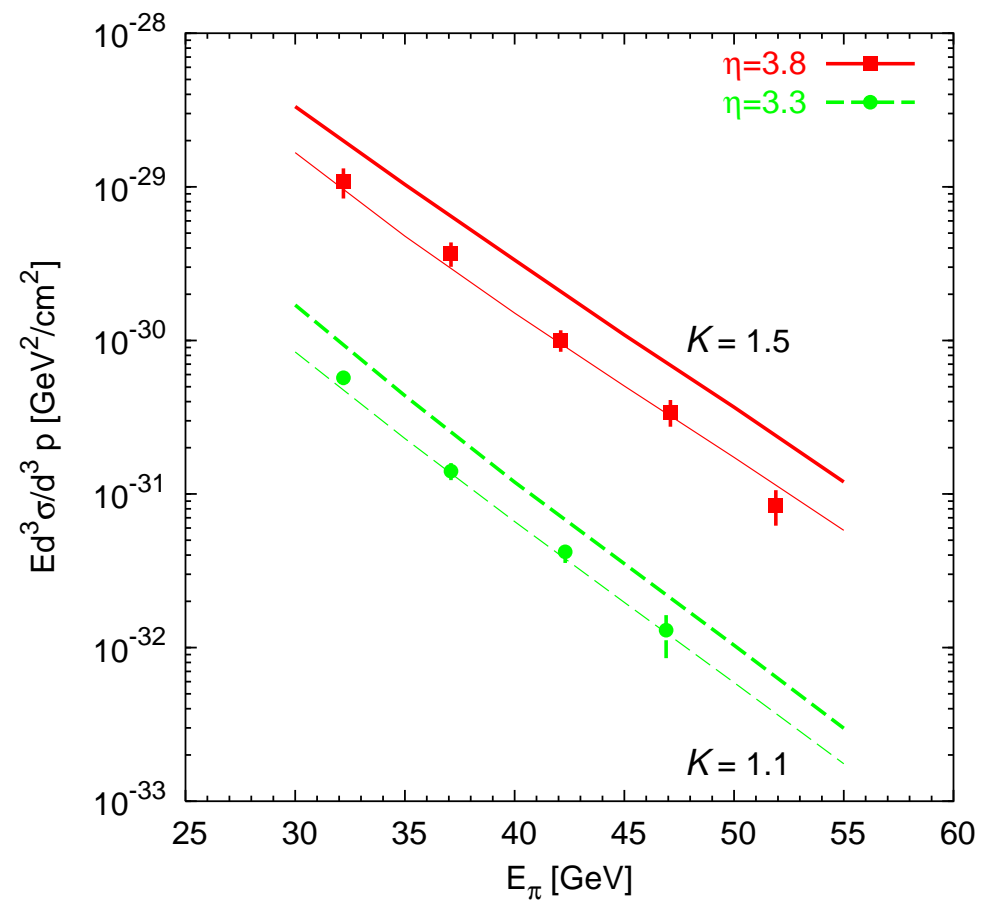

FIG. 13: Invariant differential cross section for inclusive $\pi^{0}$ production in $p p$ collisions, at $\sqrt{s}=200 \mathrm{GeV}$ and for two values of the pseudo-rapidity, $\eta$, as a function of the pion energy $E_{\pi}$. We use the parameterization MRST01 [25] for the unpolarized parton distributions, with $1 / \beta=0.8 \mathrm{GeV} / c$, and the parameterization KKP [40] for the unpolarized fragmentation functions, with $\beta^{\prime}$ given in Eq. (24) (thick lines). For comparison, the results in collinear partonic configuration (thin lines) are also shown. $K$-factors for the two cases are shown. Data are from [46]. 


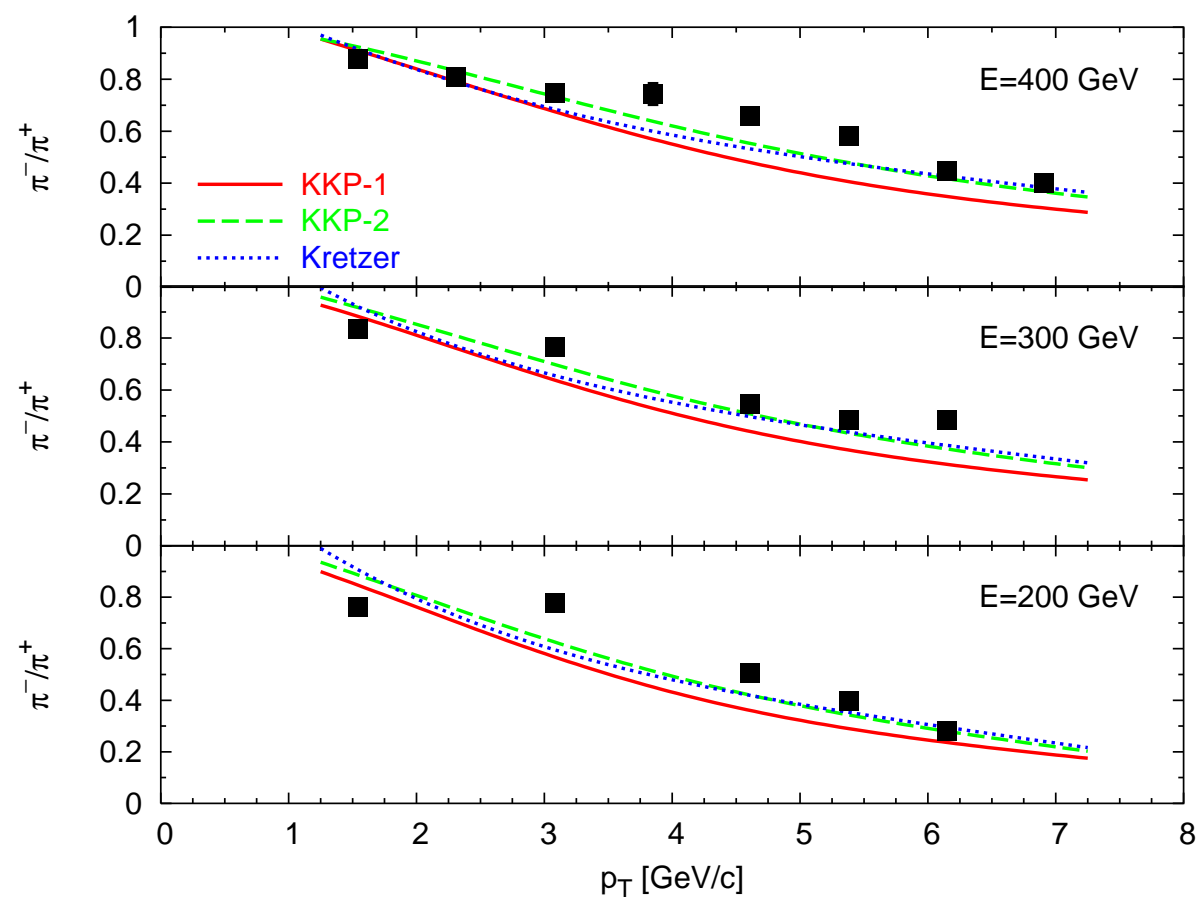

FIG. 14: Ratios of invariant differential cross sections for inclusive charged pion production in $p p$ collisions, at different LAB energies and fixed scattering angle $\theta=77(\mathrm{mrad})$, as a function of $p_{T}$. We use the parameterization MRST01 [25] for the unpolarized parton distributions, with $1 / \beta=0.8 \mathrm{GeV} / c$, and three different sets for charged pion FF's with the corresponding $\beta^{\prime}$ parameters as in Eq. (24) and (25) (see text). Data are from [48].

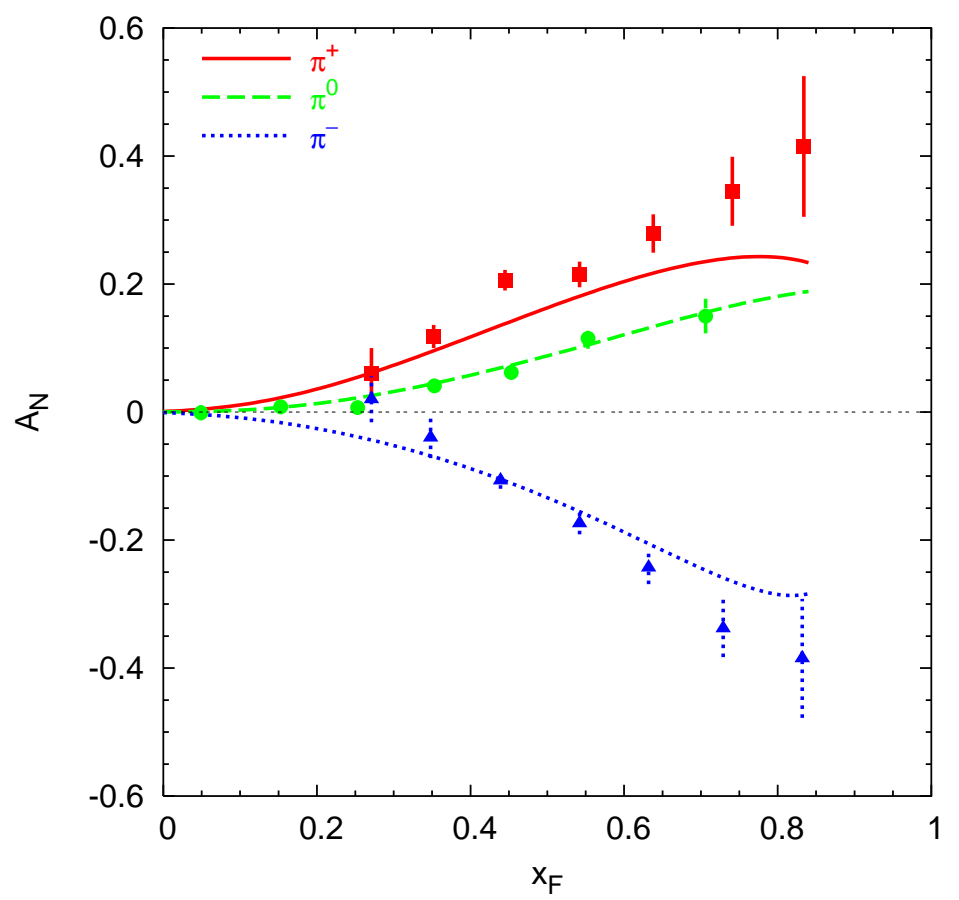

FIG. 15: $A_{N}$ for inclusive pion production in $p p$ collisions, at $\sqrt{s}=19.4 \mathrm{GeV}$ and fixed $p_{T}=1.5 \mathrm{GeV} / c$, as a function of $x_{F}$. The parameterization MRST01 [25] for the unpolarized parton distributions is used; fragmentation function set is from [47]. For the Sivers function, see Eq.s (41) and (43), parameters are given in Eq. (46), with $1 / \beta=0.8 \mathrm{GeV} / c$ and $r=0.7$. Data are from [45]. 


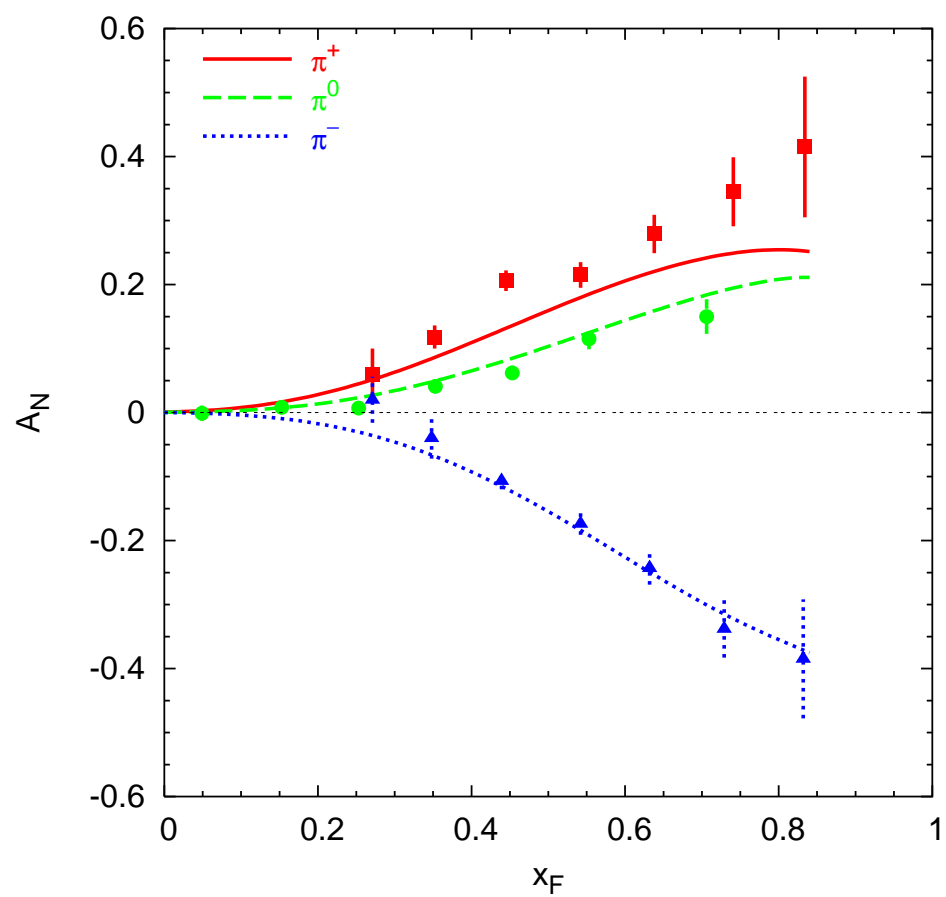

FIG. 16: $A_{N}$ for inclusive pion production in $p p$ collisions, at $\sqrt{s}=19.4 \mathrm{GeV}$ and fixed $p_{T}=1.5 \mathrm{GeV} / c$, as a function of $x_{F}$. The parameterization MRST01 [25] for the unpolarized parton distributions is used; fragmentation function set is KKP-1 (see Section II C 2). For the Sivers function, see Eq.s (41) and (43), parameters are given in Eq. (47), with $1 / \beta=0.8 \mathrm{GeV} / c$ and $r=0.7$. Data are from [45].

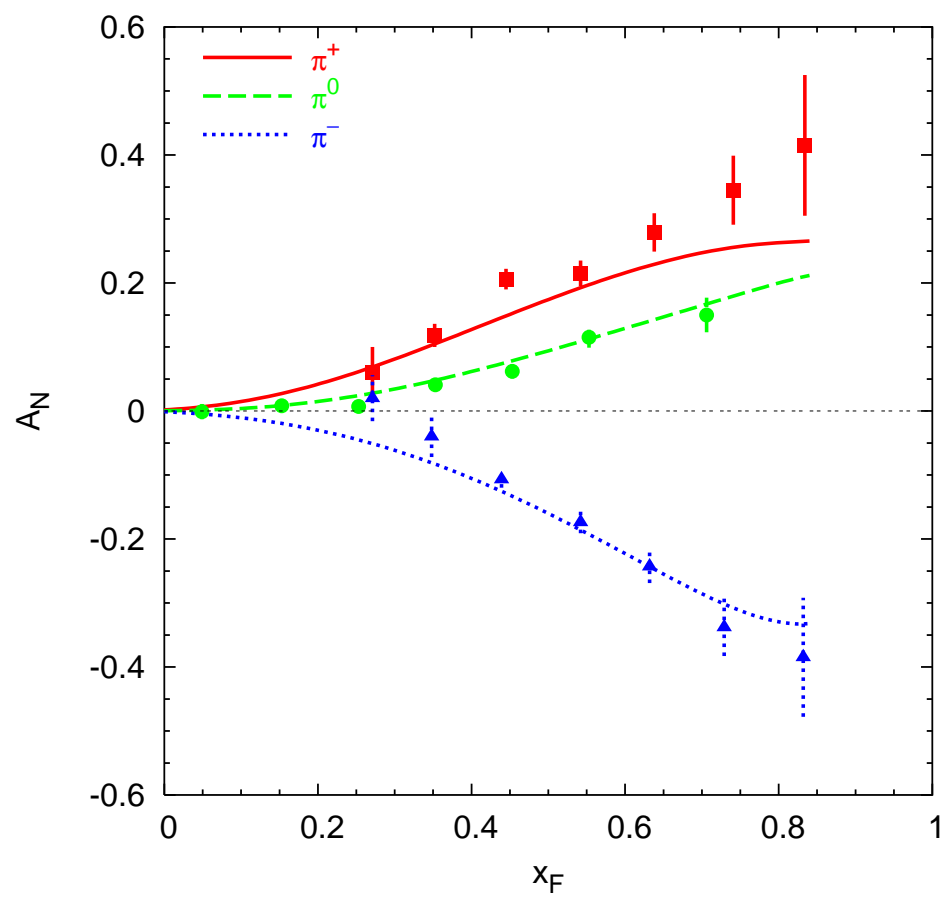

FIG. 17: $A_{N}$ for inclusive pion production in $p p$ collisions, at $\sqrt{s}=19.4 \mathrm{GeV}$ and fixed $p_{T}=1.5 \mathrm{GeV} / c$, as a function of $x_{F}$. The parameterization MRST01 [25] for the unpolarized parton distributions is used; fragmentation function set is KKP-2 (see Section II C 2). For the Sivers function, see Eq.s (41) and (43), parameters are given in Eq. (48), with $1 / \beta=0.8 \mathrm{GeV} / c$ and $r=0.7$. Data are from [45]. 


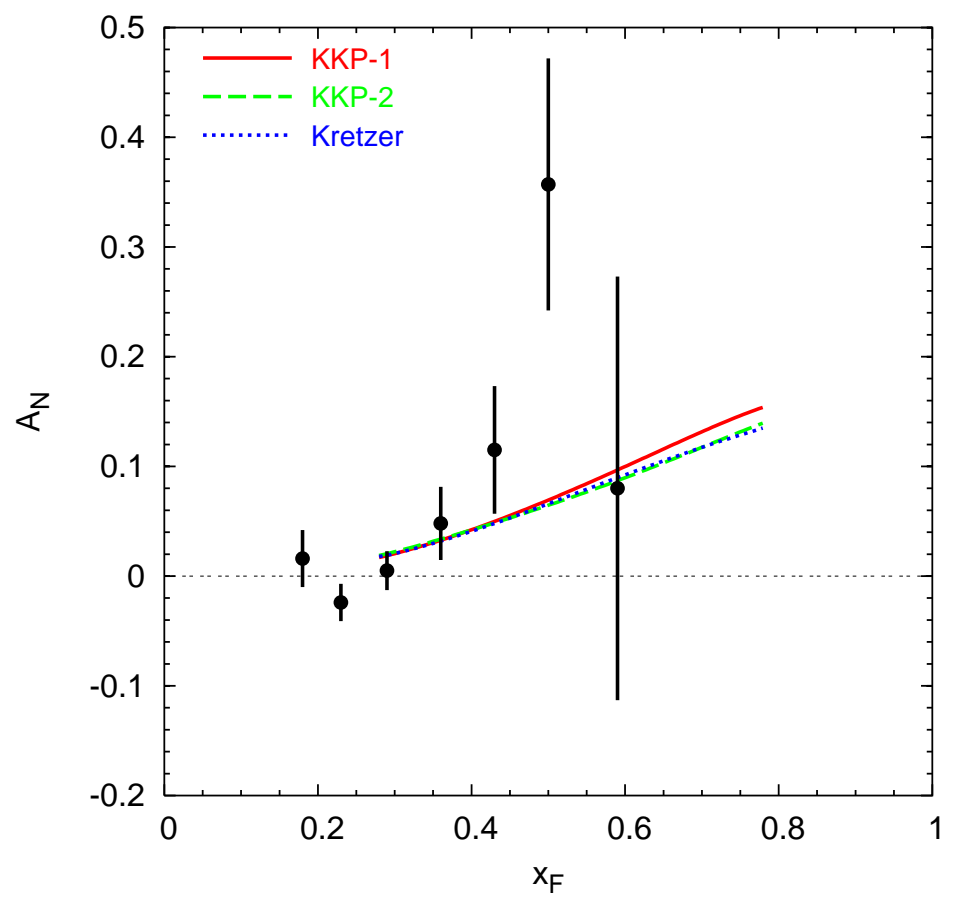

FIG. 18: $A_{N}$ for inclusive $\pi^{0}$ production in $p p$ collisions, at $\sqrt{s}=200 \mathrm{GeV}$ and fixed pseudo-rapidity $\eta=3.8$, as a function of $x_{F}$. The parameterization MRST01 [25] for the unpolarized parton distributions is used. Curves are for different fragmentation function sets and corresponding Sivers function parameterizations (see text). Data are from [54].

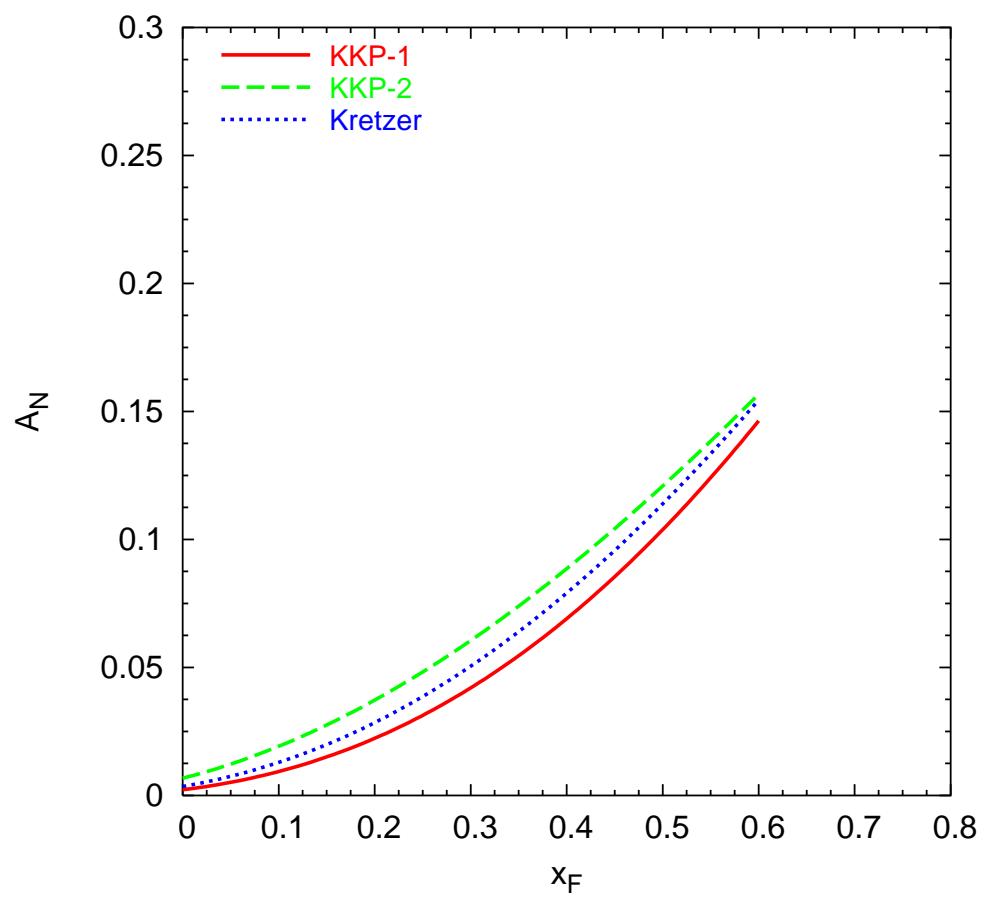

FIG. 19: $A_{N}$ for inclusive photon production in $p p$ collisions, at $\sqrt{s}=19.4 \mathrm{GeV}$ and fixed $p_{T}=2.7 \mathrm{GeV} / c$, as a function of $x_{F}$. The parameterization MRST01 [25] for the unpolarized parton distributions is used. Curves correspond to different Sivers function parameterization sets (see text). 


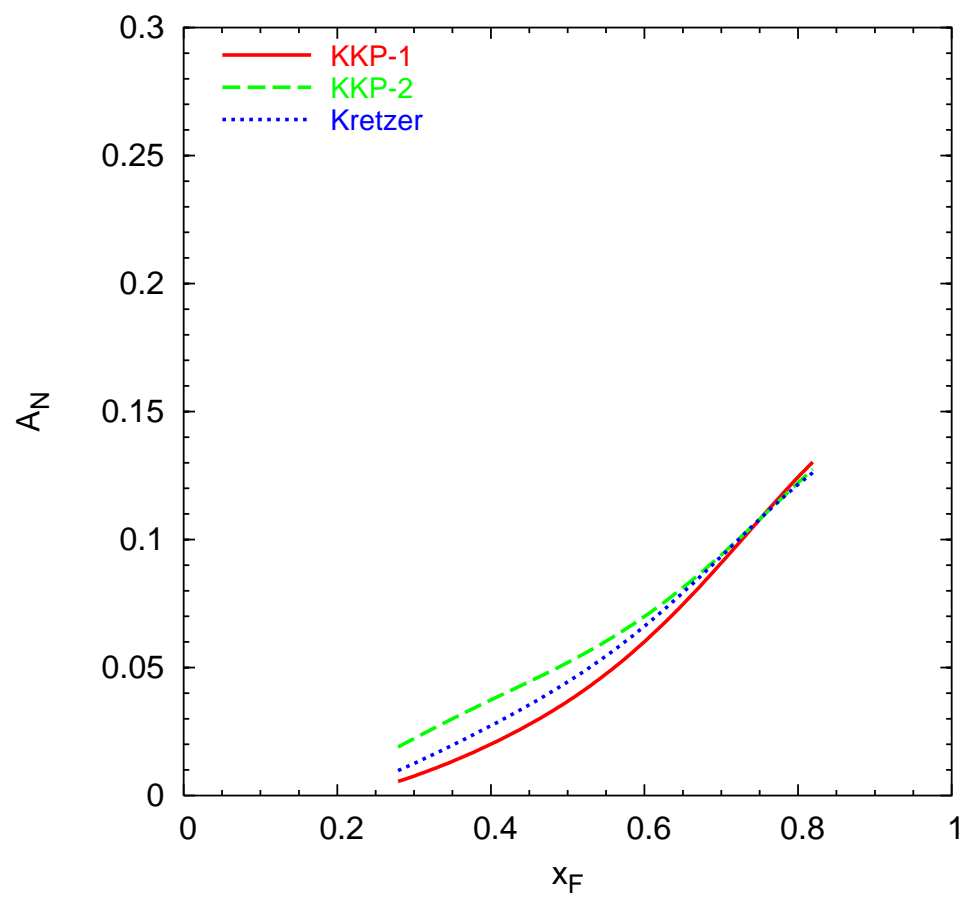

FIG. 20: $A_{N}$ for inclusive photon production in $p p$ collisions, at $\sqrt{s}=200 \mathrm{GeV}$ and fixed rapidity $y=3.8$, as a function of $x_{F}$. The parameterization MRST01 [25] for the unpolarized parton distributions is used. Curves correspond to different Sivers function parameterization sets (see text). 